\title{
Time Delay Estimation in Room Acoustic Environments: An Overview
}

\author{
Jingdong Chen, ${ }^{1}$ Jacob Benesty, ${ }^{2}$ and Yiteng (Arden) Huang ${ }^{1}$ \\ ${ }^{1}$ Bell Laboratories, Lucent Technologies, Murray Hill, NJ 07974, USA \\ ${ }^{2}$ INRS-EMT, Université du Québec, 800 de la Gauchetière Ouest, Suite 6900, Montréal, Québec, Canada H5A 1 K6
}

Received 31 January 2005; Revised 6 September 2005; Accepted 26 September 2005

Time delay estimation has been a research topic of significant practical importance in many fields (radar, sonar, seismology, geophysics, ultrasonics, hands-free communications, etc.). It is a first stage that feeds into subsequent processing blocks for identifying, localizing, and tracking radiating sources. This area has made remarkable advances in the past few decades, and is continuing to progress, with an aim to create processors that are tolerant to both noise and reverberation. This paper presents a systematic overview of the state-of-the-art of time-delay-estimation algorithms ranging from the simple cross-correlation method to the advanced blind channel identification based techniques. We discuss the pros and cons of each individual algorithm, and outline their inherent relationships. We also provide experimental results to illustrate their performance differences in room acoustic environments where reverberation and noise are commonly encountered.

Copyright ( 2006 Hindawi Publishing Corporation. All rights reserved.

\section{INTRODUCTION}

Time delay estimation (TDE), which serves as the first stage that feeds into subsequent processing blocks of a system to detect, identify, and locate radiating sources, has plenty of applications in fields as diverse as radar, sonar, seismology, geophysics, ultrasonics, and communications. It has attracted a considerable amount of research attention, ever since sensor arrays were introduced to measure a propagating wavefield.

Depending on the nature of its application, TDE can be dichotomized into two broad categories, namely, the time of arrival (TOA) estimation [1-4] and the time difference of arrival (TDOA) estimation [5-8]. The former aims at measuring the time delay between the transmission of a pulse signal and the reception of its echo, which is often of primary interest to an active system such as radar and active sonar; while the latter, as its name indicates, endeavors to determine the travel time of a wavefront between two spatially separated receiving sensors, which is often of concern to a passive system such as passive sonars and microphone array systems. Although there exists intrinsic relationship between the TOA and TDOA estimation, their essential difference is literally profound. In the former case, the "clean" reference signal, that is, the transmitted signal, is known, such that the time delay estimate can be obtained based on a single sensor generally using the matched filter approach. On the contrary, in the latter, no such explicit reference signal is available, and the delay estimate is often acquired by comparing the signals received at two (or more) spatially separated sensors. This paper deals with TDE, with its emphasis on the TDOA estimation. From now on, we will make no distinction between TDE and TDOA estimation unless necessary.

The estimation of TDOA would be an easy task if the two received signals were merely a delayed and scaled version of each other. In reality, however, the source signal is generally immersed in ambient noise since we are living in a natural environment where the existence of noise is inevitable. Furthermore, each observation signal may contain multiple attenuated and delayed replicas of the source signal due to reflections from boundaries and objects. This multipath propagation effect introduces echoes and spectral distortions into the observation signal, termed as reverberation, which severely deteriorates the source signal. In addition, the source of the wavefront may also move from time to time, resulting in a changing time delay. All these factors make time delay estimation a complicated and challenging problem. Over the past few decades, researchers have approached such a problem by exploiting different facets of the received signals. $\mathrm{Nu}-$ merous algorithms have been developed, and they can be categorized from the following points of view:

(i) the number of sources in the wavefield, that is, singlesource TDE techniques $[5,9]$ and the multiple-source TDE techniques $[10,11]$; 
(ii) how the propagation condition is modeled, that is, the ideal single-path propagation model [5], the multipath propagation model [12-14], and the reverberation model [15-17];

(iii) what analysis tools are employed, for example, generalized cross-correlation (GCC) method [5, 18-22], higher-order-statistics-(HOS) based approaches [23, 24 , and blind channel identification based algorithms $[15,25]$;

(iv) how the delay estimate is updated, that is, non-adaptive and adaptive approaches [26-30].

These methods were experimented with a certain success in various applications. However, the tolerance of TDE with respect to distortion (especially to reverberation) is still an open problem. A great deal of efforts have been made to improve the robustness of TDE techniques over the past few years. By and large, the improvements are achieved through three different ways. The first is to incorporate some a priori knowledge about the distortion sources into the GCC method to ameliorate its performance. The second is to use multiple (more than two) sensors and take advantage of the redundancy to enhance the delay estimate between the two selected sensors. The third is to take into account of reverberation in the signal model and exploit the advanced system identification techniques to improve TDE. This paper attempts to summarize these efforts, and review the state of the art, the critical techniques, and the recent advances which have significantly improved performance of time delay estimation in adverse environments. We discuss the pros and cons of each individual algorithm, and outline the relationships across different algorithms. We also provide experimental results to illustrate their performance in room acoustic environments where reverberation, noise, and interference are commonly encountered.

\section{SIGNAL MODELS FOR TDE}

Before discussing the TDE algorithms, we present mathematical models that can be employed to describe an acoustic environment for the TDE problem. Such a system modeling will, on the one hand, help us better understand the problem, and on the other hand, form a basis for discussion and analysis of various algorithms. Principally, three signal models have been used in the literature of TDE. They are the ideal single-path propagation model, the multipath model, and the reverberation model, respectively.

\subsection{Ideal propagation model}

Suppose that we have an array consisting of $N$ receivers, the ideal propagation model assumes that the signal acquired by each sensor is a delayed and attenuated version of the original source signal plus some additive noise. In a mathematical form, the received signals are expressed as

$$
x_{n}[k]=\alpha_{n} s\left[k-t-f_{n}(\tau)\right]+w_{n}[k],
$$

where $\alpha_{n}, n=0,1,2, \ldots, N-1$, are the attenuation factors due to propagation effects, $s(k)$ is the unknown source signal, $t$ is the propagation time from the unknown source to sensor $0, w_{n}[k]$ is an additive noise signal at the $n$th microphone, $\tau$ is the relative delay between microphones 0 and 1 , and $f_{n}(\tau)$ is the relative delay between microphones 0 and $n$ with $f_{0}(\tau)=$ 0 and $f_{1}(\tau)=\tau$. For $n=2, \ldots, N-1$, the function $f_{n}$ depends not only on $\tau$ but also on the microphone array geometry. For example, in the far-field case (plane wave propagation), for a linear and equispaced array, we have

$$
f_{n}(\tau)=n \tau, \quad n=2, \ldots, N-1,
$$

and for a linear but nonequispaced array, we have

$$
f_{n}(\tau)=\frac{\sum_{i=0}^{n-1} d_{i}}{d_{0}} \tau, \quad n=2, \ldots, N-1,
$$

where $d_{i}$ is the distance between microphones $i$ and $i+1$, $i=0,1,2, \ldots, N-2$. In the near-field case, $f_{n}$ depends also on the position of the source. Also note that $f_{n}(\tau)$ can be a nonlinear function of $\tau$ for a nonlinear array geometry, even in the far-field case (e.g., 3 equilateral sensors). In general $\tau$ is not known, but the geometry of the array is known such that the mathematical formulation of $f_{n}(\tau)$ is well defined or given. It is further assumed that $s[k]$ is reasonably broadband and $w_{n}[k]$ is a zero-mean Gaussian random process that is uncorrelated with both the source signal and the noise signals at other sensors. For this model, the TDE problem is formulated to determine an estimate $\hat{\tau}$ of the true time delay $\tau$ using a set of finite observation samples.

\subsection{Multipath model}

The ideal propagation model takes only into account the direct-path signal. In many situations, however, each sensor receives multiple delayed and attenuated replicas of the source signal due to reflections of the wavefront from boundaries and objects in addition to the direct-path signal. This so-called multipath effect has been intensively studied in the literature $[13,14,31,32]$. In this case, the received signals are often described mathematically as

$x_{n}[k]=\sum_{m=1}^{M} \alpha_{n m} s\left[k-t-\tau_{n m}\right]+w_{n}[k], \quad n=0,1, \ldots, N-1$,

where $\alpha_{n m}$ is the attenuation factor from the unknown source to the $n$th sensor via the $m$ th path, $t$ is the propagation time from the source to sensor 0 via direct path, $\tau_{n m}$ is the relative delay between sensor $n$ and sensor 0 for path $m$ with $\tau_{01}=0, M$ is the number of different paths, and $w_{n}[k]$ is stationary Gaussian noise and assumed to be uncorrelated with both the source signal and the noise signals observed at other sensors. This model is widely adopted in the oceanic propagation environments as illustrated in Figure 1, where each sensor receives not only the direct path signal, but reflections from both the sea surface and the sea bottom as well $[33,34]$. The primary interest of the TDE problem for this model is to measure $\tau_{n 1}, n=1, \ldots, N-1$, which is the TDOA between sensor $n$ and sensor 0 via direct path. 


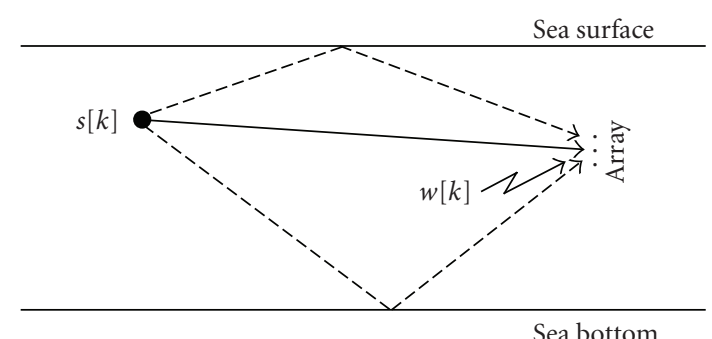

FIgURE 1: Illustration of the signal model in a multipath environment.

\subsection{Reverberation model}

The multipath model is valid for some but not all environments [35]. In addition, if there are many different paths, that is, $M$ is large, it is difficult to estimate all $\tau_{n m}$ 's in (4). Recently, a more realistic reverberation model has been used to describe the TDE problem in a room environment where each sensor often receives a large number of echoes due to reflections of the wavefront from objects and room boundaries such as walls, ceiling, and floor $[15,36,37]$. In addition, reflections can occur several times before a signal reaches the array, as shown in Figure 2. In this model, the received signals are expressed as

$$
x_{n}[k]=h_{n} * s[k]+w_{n}[k],
$$

where $*$ denotes convolution, $h_{n}$ is the channel impulse response between the source and the $n$th sensor, and again we assume that $s[n]$ is reasonably broadband and $w_{n}[k]$ is uncorrelated with $s[k]$ and the noise signals at other sensors. In a vector-matrix form, the signal model (5) can be rewritten as

$$
x_{n}[k]=\mathbf{h}_{n}^{T} \mathbf{s}[k]+w_{n}[k], \quad n=0,1, \ldots, N-1,
$$

where

$$
\begin{gathered}
\mathbf{h}_{n}=\left[\begin{array}{llll}
h_{n, 0} & h_{n, 1} & \cdots & h_{n, L-1}
\end{array}\right]^{T}, \\
\mathbf{s}[k]=\left[\begin{array}{llll}
s[k] & s[k-1] & \cdots & s[k-L+1]
\end{array}\right]^{T},
\end{gathered}
$$

and $L$ is the length of the longest channel impulse responses among $N$ channels.

As seen, no time delay is explicitly expressed in (5), hence there is no plain solution to the TDE problem with the reverberation model. In this case, TDE is often achieved in two steps. The first step is to estimate the $N$ channel impulse responses from the source to the $N$ receivers. Once the channel impulse responses are measured, the TDOA information between any two receivers is obtained by identifying the two direct paths $[15,16,38,39]$. Since we do not have any a priori knowledge about the source signal and the only information that can be accessed is the observation data, channel impulse responses have to be estimated in a blind manner. However, blind channel identification is a very challenging problem, particularly in room acoustic environments where channel impulse responses are usually very long.

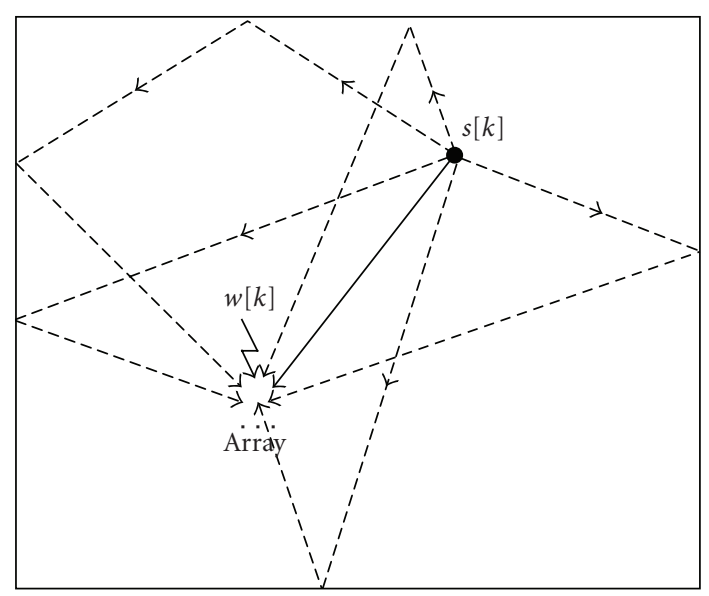

FIGURE 2: Illustration of the signal model in a reverberant environment.

\section{TDE ALGORITHMS}

Various TDE algorithms were developed in the literature. In this section, we brief some critical techniques. Some of them have already been widely used, while others may not be popular with existing systems, but have the great potential for use in future ones.

\subsection{Cross-correlation method}

The cross-correlation (CC) method is the most straightforward and the earliest developed TDE algorithm, which is formulated based on the single-path propagation model given in (1) with only two receivers, that is, $N=2$. Suppose that we have a block of observation signals at time instant $k$,

$$
\begin{aligned}
\mathbf{x}_{n}[k] & =\left[x_{n}[0], x_{n}[1], \ldots, x_{n}[l], \ldots, x_{n}[K-1]\right]^{T} \\
& =\left[x_{n}[k], x_{n}[k+1], \ldots, x_{n}[k+K-1]\right]^{T},
\end{aligned}
$$

where $n=0,1$ and $K$ is the block size, then the delay estimate with the CC method is obtained as the lag time that maximizes the cross-correlation function (CCF) between two observation signals, that is,

$$
\hat{\tau}_{\mathrm{CC}}=\arg \max _{m} \Psi_{\mathrm{CC}}[m],
$$

where

$$
\Psi_{\mathrm{CC}}[m]=E\left\{x_{0}[l] x_{1}[l+m]\right\}
$$

is the CCF between $x_{0}[l]$ and $x_{1}[l], E\{\cdot\}$ stands for the mathematical expectation, $\hat{\tau}_{\mathrm{CC}}$ is an estimate of the true delay $\tau$, $m \in\left[-\tau_{\max }, \tau_{\max }\right]$, and $\tau_{\max }$ is the maximum possible delay. In digital implementation of (9), some approximations are required because the CCF is not known and must be estimated. A normal practice is to replace the CCF defined in 
(10) by its time-averaged estimate, that is,

$$
\hat{\Psi}_{\mathrm{CC}}[m]= \begin{cases}\frac{1}{K} \sum_{l=0}^{K-m-1} x_{0}[l] x_{1}[l+m], & m \geq 0, \\ \frac{1}{K} \sum_{l=-m}^{K-1} x_{0}[l] x_{1}[l+m], & m<0 .\end{cases}
$$

A similar method, formulated from the average-magnitude-difference function (AMDF), was also investigated in the literature [40], where the TDE becomes to identify the minimum of AMDF, that is,

$$
\hat{\tau}_{\mathrm{AMDF}}=\arg \min _{m} \hat{\Psi}_{\mathrm{AMDF}}[m]
$$

where

$$
\hat{\Psi}_{\mathrm{AMDF}}[m]= \begin{cases}\frac{1}{K} \sum_{l=0}^{K-m-1}\left|x_{0}[l]-x_{1}[l+m]\right|, & m \geq 0, \\ \frac{1}{K} \sum_{l=-m}^{K-1}\left|x_{0}[l]-x_{1}[l+m]\right|, & m<0,\end{cases}
$$

is the AMDF between $x_{0}[l]$ and $x_{1}[l]$. It has been shown that $[41,42]$

$$
E\left\{\hat{\Psi}_{\mathrm{AMDF}}[m]\right\}=\sqrt{\frac{2}{\pi}\left[E\left\{x_{0}^{2}[l]\right\}+E\left\{x_{1}^{2}[l]\right\}-2 E\left\{\hat{\Psi}_{\mathrm{CC}}[m]\right\}\right]} .
$$

There are three terms in the brackets under the square root of (14): the first two are the signal energies, and the third is the expectation of CCF. The signal energy, which can be treated as a constant during the observation period, does not affect the peak position. Therefore, statistically, searching the minimum of the AMDF is same as finding the maximum of the CCF between two observation signals. As a result, the AMDF approach should exhibit a similar performance to the CC method from a statistical point of view [43].

\subsection{Generalized cross-correlation method}

The generalized cross-correlation (GCC) algorithm can be treated as an improved version of the CC method. Not only does it unify various correlation-based algorithms into one general framework, but it also provides a mechanism to incorporate knowledge to improve the performance of TDE. This method has gained its great popularity since the landmark paper [5] was published by Knapp and Carter in 1976. In this framework, the delay estimate is obtained as

$$
\hat{\tau}_{\mathrm{GCC}}=\arg \max _{m} \Psi_{\mathrm{GCC}}[m],
$$

where

$$
\begin{aligned}
\Psi_{\mathrm{GCC}}[m] & =\sum_{k^{\prime}=0}^{K^{\prime}-1} \Phi\left[k^{\prime}\right] S_{x_{0} x_{1}}\left[k^{\prime}\right] e^{j 2 \pi m k^{\prime} / K^{\prime}} \\
& =\sum_{k^{\prime}=0}^{K^{\prime}-1} \varsigma_{x_{0} x_{1}}\left[k^{\prime}\right] e^{j 2 \pi m k^{\prime} / K^{\prime}}
\end{aligned}
$$

is so-called generalized cross-correlation function (GCCF), $S_{x_{0} x_{1}}\left[k^{\prime}\right]=E\left\{X_{0}\left[k^{\prime}\right] X_{1}^{*}\left[k^{\prime}\right]\right\}$ is the cross-spectrum, $(\cdot)^{*}$ denotes the complex conjugate operator, $X_{n}\left[k^{\prime}\right]$ is the discrete Fourier transform (DFT) of $\mathbf{x}_{n}[k], \Phi\left[k^{\prime}\right]$ is a weighting function (sometimes called a prefilter), $K^{\prime}$ is the length of the DFT, and $\varsigma_{x_{0} x_{1}}\left[k^{\prime}\right]=\Phi\left[k^{\prime}\right] S_{x_{0} x_{1}}\left[k^{\prime}\right]$ is the weighted crossspectrum. In a practical system, the cross-spectrum $S_{x_{0} x_{1}}\left[k^{\prime}\right]$ has to be estimated, which is normally achieved by replacing the expected value by its instantaneous value, that is, $\widehat{S}_{x_{0} x_{1}}\left[k^{\prime}\right]=X_{0}\left[k^{\prime}\right] X_{1}^{*}\left[k^{\prime}\right]$.

There is a number of member algorithms in the GCC family depending on how the weighting function $\Phi\left[k^{\prime}\right]$ is selected. Commonly used weighting functions include the constant weighting (in this case, the GCC becomes a frequencydomain implementation of the cross-correlation method shown in (9)), the smoothed coherence transform (SCOT) [44], the Roth processor [45], the Echart filter [5], the phase transform (PHAT), the maximum-likelihood (ML) processor [5], the Hassab-Boucher transform [18], and so forth. Combination of some of these functions is also reported in use [46].

Different weighting functions possess different properties. For example, the PHAT algorithm uses $\Phi_{\text {PHAT }}\left[k^{\prime}\right]=$ $1 /\left|S_{x_{0} x_{1}}\left[k^{\prime}\right]\right|$. Substituting $\Phi_{\text {PHAT }}\left[k^{\prime}\right]$ into (15) and neglecting noise effects, one can readily deduce that the weighted crossspectrum is free from the source signal and depends only on the channel responses. Consequently the PHAT algorithm performs more consistently than many other GCC members when the characteristics of the source signal change over time. It is also observed that the PHAT algorithm is more immune to reverberation than many other cross-correlationbased methods. Another example is the ML processor with which the delay estimate obtained in the ideal propagation situation is optimal from a statistical point of view since the estimation variance can achieve the Cramèr-Rao lower bound (CRLB). It should be pointed out that in order for the ML processor to achieve the optimal performance, the observation sample space has to be large enough; the environments should be free of reverberation; the delay has to be constant; and the observation signals should be stationary processes. In addition, the spectra of noise signals have to be known a priori. If any of these conditions does satisfy, the ML algorithm will then become suboptimal, like other GCC members.

\subsection{LMS-type adaptive TDE algorithm}

This method, also based on the ideal propagation model with two sensors, was proposed by Reed et al. in 1981 [26]. It has been intensively investigated in the literature since 
then [28-30, 47]. Different from the cross-correlation-based approaches, this algorithm achieves time delay by minimizing the mean-square error between $x_{0}[k]$ and a filtered (FIR filter) version of $x_{1}[k]$, and the delay estimate is obtained as the lag time associated with the largest component of the FIR filter. If we define a signal vector of $x_{1}[k]$ at time instant $k$ as

$$
\begin{aligned}
\mathbf{x}_{1}[k]=[ & x_{1}[k-L], x_{1}[k-L+1], \ldots, x_{1}[k], \\
& \left.x_{1}[k+1], \ldots, x_{1}[k+L]\right]^{T}
\end{aligned}
$$

and an FIR filter of length $2 L+1$ as

$$
\mathbf{h}[k]=\left[h_{0}, h_{1}, \ldots, h_{l}, h_{l+1}, \ldots, h_{2 L}\right]^{T},
$$

where $L$ is the maximum possible time delay, then an error signal can be formulated as

$$
e[k]=x_{0}[k]-\mathbf{h}^{T}[k] \mathbf{x}_{1}[k] .
$$

An estimate of $\mathbf{h}[k]$ can be achieved by minimizing $E\left\{e^{2}[k]\right\}$ using either a batch or an adaptive algorithm. For example, with the least-mean-square (LMS) adaptive algorithm, $\mathbf{h}[k]$ can be estimated through

$$
\mathbf{h}[k+1]=\mathbf{h}[k]+\mu e[k] \mathbf{x}_{1}[k],
$$

where $\mu$ is a small positive adaptation step size. Given this estimate of $\mathbf{h}[k]$, the delay estimate can be determined as

$$
\hat{\tau}_{\text {LMS }}=\arg \max _{l}\left|h_{l}\right|-L .
$$

Other adaptive algorithms [48] can also be used, which may lead to a better performance.

\subsection{Fusion algorithm based on multiple sensor pairs}

The GCC framework, which may yield much improvement over the traditional direct cross-correlation method if the weighting function is properly selected, still suffers significant performance degradation in adverse environments. Much attention has been paid to improving the tolerance of TDE against noise and reverberation. Besides using some a priori knowledge about the distortion sources, another way of combating noise and reverberation is through exploiting the redundant information provided by multiple sensors. To illustrate the redundancy, let us consider a three-sensor linear array, which can be partitioned into three sensor pairs. Three delay measurements can then be acquired with the observation data, that is, $\tau_{01}$ (TDOA between sensor 0 and sensor 1 ), $\tau_{12}$ (TDOA between sensor 1 and sensor 2 ), and $\tau_{02}$ (TDOA between sensor 0 and sensor 2). Apparently, these three delays are not independent. As a matter of fact, if the source is located in the far field, it is easily seen that $\tau_{02}=\tau_{01}+\tau_{12}$. Such a relation was exploited in [49] to formulate a twostage TDE algorithm. In the preprocessing stage, three delay measurements were measured independently using the GCC method. A state equation was then formed and a Kalman filter is used in the postprocessing stage to enhance the delay estimate of $\tau_{01}$ and $\tau_{12}$. It was shown that in the far-field case, the estimation variance of $\tau_{01}$ can be reduced by a factor of 6 in low SNR $($ SNR $\rightarrow 0)$, and of 4 in high SNR $(\operatorname{SNR} \rightarrow \infty)$ conditions. More recently, several approaches based on multiple sensor pairs were developed to deal with TDE in room acoustic environments [50-52]. Different from the Kalman filter method, these approaches fuse the estimated cost functions from multiple sensor pairs before searching the time delay. We will call such a scheme as information fusion based algorithm. In general, the problem of TDE with the fusion algorithm can be formulated as

$$
\hat{\tau}_{\mathrm{FUSION}}=\arg \max _{m} \sum_{p=1}^{P} \mathcal{F}\left\{\hat{\Psi}_{p}[m]\right\}
$$

where $P$ is the total number of sensor pairs, $\hat{\Psi}_{p}[m]$ represents some delay cost function measured from the $p$ th sensor pair (it can be CCF, GCCF, AMDF, etc.), and $\mathcal{F}\{\cdot\}$ denotes some mathematical transformation, which ensures that the cost functions $\left(\hat{\Psi}_{p}[m]\right)$ for all the $P$ sensor pairs, after transformation, have their peaks due to the same source in the same location. Various methods can be formulated by selecting a different $\mathcal{F}\{\cdot\}$ or $\hat{\Psi}$. For example, if all sensor pairs are centered around a same position, by choosing $\mathcal{F}\{x\}=x$, $\hat{\Psi}[m]$ as the GCCF from the PHAT algorithm, one can readily derive the so-called synchronous adding method in [50]. We can also easily derive the consistency method in [51] and the SRP (steered response power)-PHAT algorithm in [52]. Compared with the algorithms using only two sensors, the fusion technique can usually deliver a better performance. However, its computational complexity is also more than $P$ times of the complexity of the corresponding dual-sensor technique, where $P$ is the number of sensor pairs.

\subsection{Multichannel cross-correlation algorithm}

Recently, a squared multichannel cross-correlation coefficient (MCCC) was derived from the theory of spatial linear prediction and interpolation [53]. Consider the signal model given in (1) with a total of $N$ sensors. At time instant $k$, the MCCC is defined as

$$
\varrho_{N}^{2}(k, m)=1-\frac{\operatorname{det}[\mathbf{R}(k, m)]}{\prod_{l=0}^{N-1} r_{l l}(k, m)}=1-\operatorname{det}[\widetilde{\mathbf{R}}(k, m)],
$$

where "det" stands for determinant of a matrix,

$\mathbf{R}(k, m)=\left[\begin{array}{cccc}r_{00}(k, m) & r_{01}(k, m) & \cdots & r_{0 N-1}(k, m) \\ r_{10}(k, m) & r_{11}(k, m) & \cdots & r_{1 N-1}(k, m) \\ \vdots & \ddots & \ddots & \vdots \\ r_{N-10}(k, m) & r_{N-11}(k, m) & \cdots & r_{N-1 N-1}(k, m)\end{array}\right]$,

is the signal covariance matrix,

$$
\begin{array}{r}
r_{i j}(k, m)=\sum_{p=0}^{k} \lambda^{k-p} x_{i}\left[p+f_{j}(m)\right] x_{j}\left[p+f_{i}(m)\right], \\
i, j=0,1, \ldots, N-1,
\end{array}
$$


is the cross-correlation function between $x_{i}$ and $x_{j}$ (similar as what is defined in $(11)), \lambda(0<\lambda \leq 1)$ is a forgetting factor,

$$
\begin{gathered}
\widetilde{\mathbf{R}}(k, m)=\left[\begin{array}{cccc}
1 & \rho_{01}(k, m) & \cdots & \rho_{0 N-1}(k, m) \\
\rho_{10}(k, m) & 1 & \cdots & \rho_{1 N-1}(k, m) \\
\vdots & \ddots & \ddots & \vdots \\
\rho_{N-10}(k, m) & \rho_{N-11}(k, m) & \cdots & 1
\end{array}\right], \\
\rho_{i j}(k, m)=\frac{r_{i j}(k, m)}{\sqrt{r_{i i}(k, m) r_{j j}(k, m)}}, \quad i, j=0,1, \ldots, N-1,
\end{gathered}
$$

is the cross-correlation coefficient between $x_{i}$ and $x_{j}$. With this definition, the MCCC can be estimated either in a batch mode, which operates on a block of data snapshots [53], or in a recursive way, which updates the estimate whenever a new snapshot is available [54].

Just like the cross-correlation coefficient between two signals, this definition of multichannel cross-correlation coefficient possesses quite a few good properties, and can be treated as a natural generalization of the traditional cross-correlation coefficient from the two-channel to the multichannel cases. The problem of TDE at time instant $k$, based on this new definition, can be formulated as

$$
\begin{aligned}
\hat{\tau}_{\mathrm{MCCC}} & =\arg \max _{m} \varrho_{N}^{2}(k, m) \\
& =\arg \max _{m}\{1-\operatorname{det}[\widetilde{\mathbf{R}}(k, m)]\} \\
& =\arg \min _{m}\{\operatorname{det}[\widetilde{\mathbf{R}}(m, k)]\}
\end{aligned}
$$

For the particular case where we have only two receiving sensors, it can be checked that

$$
\begin{aligned}
\hat{\tau}_{\mathrm{MCCC}} & =\arg \max _{m} \varrho_{N}^{2}(k, m) \\
& =\arg \max _{m} \rho_{01}^{2}(k, m),
\end{aligned}
$$

which is same as the cross-correlation method shown in Section 3.1. When we have more than two sensors, this method can be viewed as a natural generalization of the cross-correlation method to the multichannel case, which can take advantage of the redundancy among multiple sensors to improve the time delay estimate between two sensors. It is worth mentioning that a prewhitening process can be applied to the observation signals before delay estimation. In this case, the MCCC algorithm can be treated as a generalized version of the PHAT algorithm.

\subsection{Adaptive eigenvalue decomposition algorithm}

All the algorithms outlined in the previous sections achieve delay estimate by measuring the cross-correlation between two or among multiple channels. A common assumption with these methods is that each sensor receives only the direct-path signal. Recently, an adaptive eigenvalue decomposition (AED) algorithm was proposed to deal with TDE in room reverberant environment $[15,55]$. Unlike the crosscorrelation-based methods, this algorithm first identifies the channel impulse responses from the source to the two sensors. The delay estimate is then determined by finding the direct paths from the two measured impulse responses. Apparently, this algorithm takes fully into account the reverberation effect during time delay estimation.

For the signal model given in (5) with two sensors, if the noise term is neglected, one can easily check that

$$
x_{0}[k] * h_{1}=s[k] * h_{0} * h_{1}=x_{1}[k] * h_{0} .
$$

At time instant $k$, this relation can be rewritten in a vectormatrix form as [15]

$$
\mathbf{x}^{T}[k] \mathbf{u}=\mathbf{x}_{0}^{T}[k] \mathbf{h}_{1}-\mathbf{x}_{1}^{T}[k] \mathbf{h}_{0}=0
$$

where

$$
\begin{aligned}
& \mathbf{x}_{n}[k]=\left[\begin{array}{llll}
x_{n}[k] & x_{n}[k-1] & \cdots & x_{n}[k-L+1]
\end{array}\right]^{T}, \\
& \mathbf{x}[k]=\left[\begin{array}{ll}
\mathbf{x}_{0}^{T}[k] & \mathbf{x}_{1}^{T}[k]
\end{array}\right]^{T}, \\
& \mathbf{u}=\left[\begin{array}{ll}
\mathbf{h}_{1}^{T} & -\mathbf{h}_{0}^{T}
\end{array}\right]^{T},
\end{aligned}
$$

and $n=0,1$. Left multiplying (30) by $\mathbf{x}[n]$ and taking expectation yields

$$
\mathbf{R u}=\mathbf{0},
$$

where $\mathbf{R}=E\left\{\mathbf{x}[k] \mathbf{x}^{T}[k]\right\}$ is the covariance matrix of the sensor signals. This implies that vector $\mathbf{u}$ which consists of two impulse responses is in the null space of $\mathbf{R}$. More specifically, $\mathbf{u}$ is the eigenvector of $\mathbf{R}$ corresponding to the eigenvalue 0 . It has been shown that the two channel impulse responses (i.e., $\mathbf{h}_{0}$ and $\mathbf{h}_{1}$ ) can be uniquely determined (up to a scale and a common delay) from (32) if the following two conditions hold [56-58]:

(i) the polynomials formed from $\mathbf{h}_{0}$ and $\mathbf{h}_{1}$ (i.e., the $Z$ transforms of $\mathbf{h}_{0}$ and $\mathbf{h}_{1}$ ) are coprime, or they do not share any common zeros;

(ii) the autocorrelation matrix of the source signal $s[k]$, that is, $\mathbf{R}_{s s}=E\left\{\mathbf{s}[k] \mathbf{s}^{T}[k]\right\}$, is of full rank.

See $[56,59]$ for a detailed description about the necessary and sufficient conditions for the identifiability. Note that the scale and common-delay ambiguities of blind identification techniques does not affect the problem of TDE.

When an independent white noise signal is present on each sensor, it will regularize the covariance matrix; as a consequence, $\mathbf{R}$ does not have a zero eigenvalue anymore. In such a case, an estimate of the impulse responses can be achieved through the following algorithm, which is an adaptive way to find the eigenvector associated with the smallest eigenvalue 
of R [15]:

$$
\widehat{\mathbf{u}}[k+1]=\frac{\widehat{\mathbf{u}}[k]-\mu e[k] \mathbf{x}[k]}{\|\hat{\mathbf{u}}[k]-\mu e[k] \mathbf{x}[k]\|},
$$

with the constraint that $\|\hat{\mathbf{u}}[k]\|=1$, where

$$
e[k]=\widehat{\mathbf{u}}^{T}[k] \mathbf{x}[k]
$$

is an error signal, $\|\cdot\|$ denotes the $l_{2}$ norm of a vector or matrix, and $\mu$, the adaptation step, is a positive constant.

With the identified impulse responses $\hat{\mathbf{h}}_{0}$ and $\hat{\mathbf{h}}_{1}$, the time delay estimate is determined as the difference between two direct paths, that is,

$$
\hat{\tau}_{\mathrm{AED}}=\arg \max _{l}\left|\hat{h}_{1, l}\right|-\arg \max _{l}\left|\hat{h}_{0, l}\right| .
$$

\subsection{Adaptive multichannel time delay estimation}

In the AED algorithm, the delay estimate is obtained by blindly identifying two channel impulse responses. It requires that the two channels do not share any common zeros, which is usually true for systems with short impulse responses. In many application scenarios such as room acoustic environments, however, the channel impulse response from the source to the microphone sensor could be very long, depending on the reverberation condition. As the length of the two impulse responses becomes longer, the probability for them not sharing common zeros will become lower and the AED algorithm often fails when a zero is shared between two channels or some zeros of the two channels are close. One way to overcome this problem is to employ more channels in the system, since it would be less likely for all channels to share a common zero when the number of sensors is large. This idea leads to an adaptive multichannel (AMC) time delay estimation approach based on a blind channel identification technique [39].

Considering the reverberation model in (5), we can define a cost function among all the $N$ channels, at time instant $k+1$, as

$$
J[k+1]=\sum_{i=0}^{N-2} \sum_{j=i+1}^{N-1} e_{i j}^{2}[k+1]
$$

where

$$
\begin{array}{r}
e_{i j}[k+1]=\frac{\mathbf{x}_{i}^{T}[k+1] \hat{\mathbf{h}}_{j}[k]-\mathbf{x}_{j}^{T}[k+1] \hat{\mathbf{h}}_{i}[k]}{\|\hat{\mathbf{h}}[k]\|}, \\
i, j=0,1, \ldots, N-1,
\end{array}
$$

is an error signal between sensor $i$ and sensor $j$ at time $k+1$, $\widehat{\mathbf{h}}_{n}[k]$ is the modeling filter of $\mathbf{h}_{n}[k]$, and

$$
\hat{\mathbf{h}}[k]=\left[\begin{array}{llll}
\hat{\mathbf{h}}_{0}^{T}[k] & \hat{\mathbf{h}}_{1}^{T}[k] & \cdots & \hat{\mathbf{h}}_{N-1}^{T}[k]
\end{array}\right]^{T} .
$$

It follows immediately that various adaptive algorithms can be used to achieve an estimate of $\hat{\mathbf{h}}[k]$, by minimizing $J[k+1]$.
For example, a multichannel LMS (MCLMS) algorithm was derived in [60], which updates $\hat{\mathbf{h}}$ through

$$
\widehat{\mathbf{h}}[k+1]=\frac{\hat{\mathbf{h}}[k]-2 \mu[\widetilde{\mathbf{R}}[k+1] \hat{\mathbf{h}}[k]-J[k+1] \hat{\mathbf{h}}[k]]}{\|\hat{\mathbf{h}}[k]-2 \mu[\widetilde{\mathbf{R}}[k+1] \hat{\mathbf{h}}[k]-J[k+1] \hat{\mathbf{h}}[k]]\|},
$$

where again $\mu$, the adaptation step, is a positive constant,

$$
\begin{aligned}
& \widetilde{\mathbf{R}}[k+1]= \\
& {\left[\begin{array}{cccc}
\sum_{i \neq 0} \widetilde{\mathbf{R}}_{x_{i} x_{i}}[k+1] & -\widetilde{\mathbf{R}}_{x_{1} x_{0}}[k+1] & \cdots & -\widetilde{\mathbf{R}}_{x_{N-1} x_{0}}[k+1] \\
-\widetilde{\mathbf{R}}_{x_{0} x_{1}}[k+1] & \sum_{i \neq 1} \widetilde{\mathbf{R}}_{x_{i} x_{i}}[k+1] & \cdots & -\widetilde{\mathbf{R}}_{x_{N-1} x_{1}}[k+1] \\
\vdots & \vdots & \ddots & \vdots \\
-\widetilde{\mathbf{R}}_{x_{0} x_{N-1}}[k+1] & -\widetilde{\mathbf{R}}_{x_{1} x_{N-1}}[k+1] & \cdots & \sum_{i \neq N-1} \widetilde{\mathbf{R}}_{x_{i} x_{i}}[k+1]
\end{array}\right],} \\
& \widetilde{\mathbf{R}}_{x_{i} x_{j}}[k+1]=\mathbf{x}_{i}[k+1] \mathbf{x}_{j}^{T}[k+1], \quad i, j=0,1, \ldots, N-1 .
\end{aligned}
$$

It was shown that with this MCLMS algorithm the channel estimate can converge in mean to the true impulse responses (up to a scale and common delay). However, the convergence rate of this algorithm is normally slow. To accelerate the convergence rate, a normalized multichannel frequency-domain LMS (NMCFLMS) algorithm was developed in [25]. Different from the MCLMS method, which updates the channel estimate every snapshot, the (NMCFLMS) algorithm operates in the frequency domain on a block-by-block basis. First, the multichannel observation signals are partitioned into successive blocks. The fast Fourier transform (FFT) is then applied to each block to estimate its Fourier spectrum. The frequency-domain channel estimate is then updated using the normalized LMS algorithm. Finally, the time-domain impulse responses are obtained by applying the inverse FFT to the frequency-domain channel estimate. See Algorithm 5 for how to obtain the channel estimates and [25] for the detailed derivation of the NMCFLMS algorithm.

Once $\hat{\mathbf{h}}[k]$ is achieved (with either the MCLMS algorithm or the NMCFLMS algorithm), the time-domain estimate of impulse responses is obtained by the inverse Fourier transform, and time delay between the $i$ th and $j$ th sensors is determined as

$$
\hat{\tau}_{i j}=\arg \max _{l}\left|\hat{h}_{j, l}\right|-\arg \max _{l}\left|\hat{h}_{i, l}\right| .
$$

\section{ALGORITHM COMPLEXITY}

This section briefly compares the computational complexity of different TDE algorithms. As seen, all the algorithms estimate time-delay information in two steps. The first step involves the estimation of the cost function. The second step obtains time delay estimate by searching the extremum of the cost function. If we assume that different cost functions have the same length, it can be easily checked that all the 


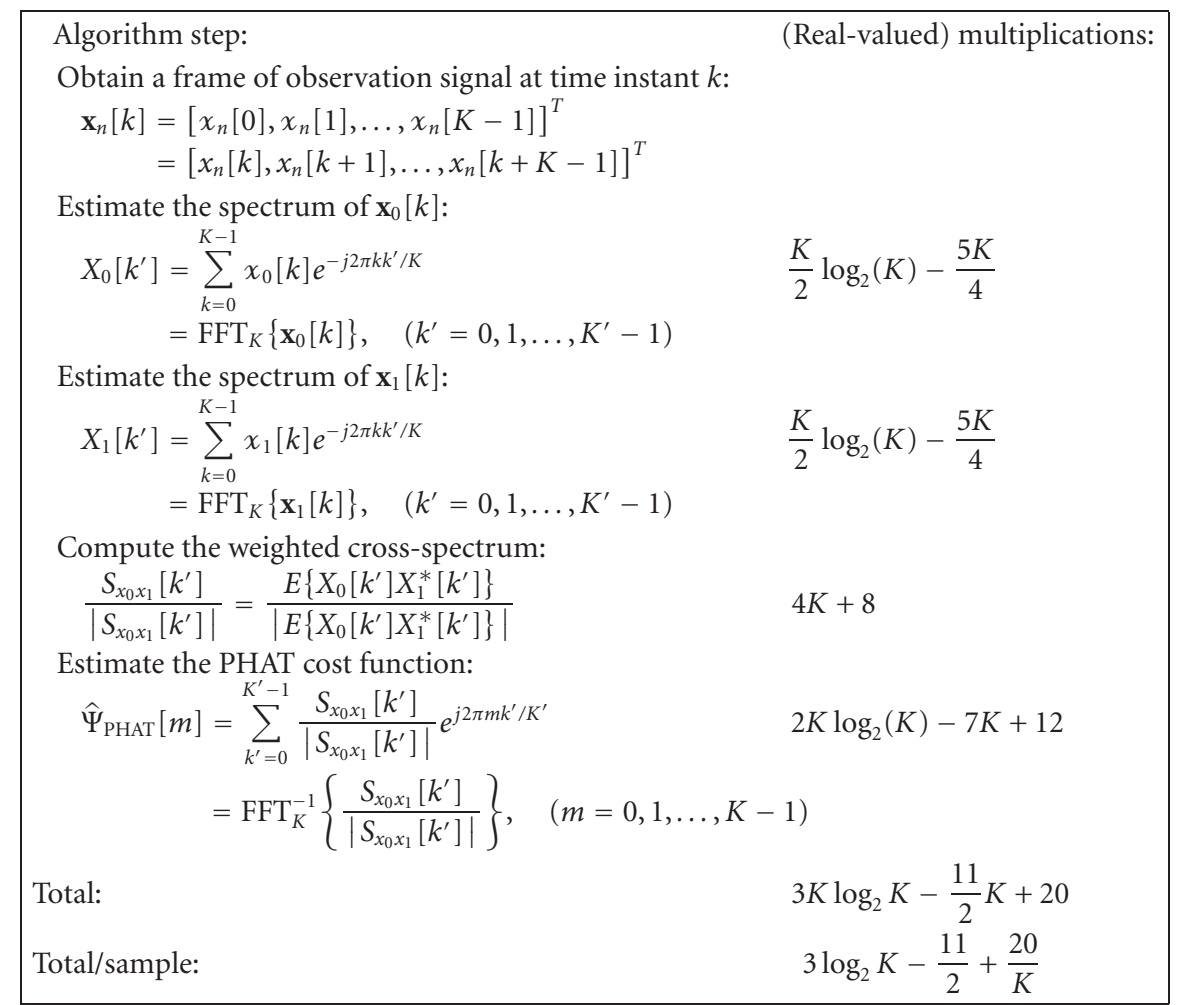

Algorithm 1: Computational complexity of the PHAT algorithm. $\operatorname{FFT}_{K}\{\cdot\}$ and $\operatorname{IFFT}_{K}^{-1}\{\cdot\}$ are $K$-point fast Fourier and inverse fast Fourier transforms, respectively. In addition, due to the symmetric property, we only need to perform $K / 2+1$ complex multiplications and divisions during computation of the weighted spectrum.

algorithms have a similar complexity in the second step. Therefore, we only compare the computational burdens required for estimating the cost function. Here the computational complexity is evaluated in terms of the number of real-valued multiplications/divisions required for the implementation of each algorithm. The number of additions/subtractions are neglected because they are much quicker to compute in most generic hardware platforms. We assume that complex-valued multiplications are transformed into real-valued multiplications. The multiplication between a real number and complex number requires 2 real-valued multiplications. The multiplication between two complex numbers needs 4 real-valued multiplications. The division between a complex number and a real number requires 2 real-valued multiplications.

As mentioned earlier, there are different member algorithms in the GCC family. Each involves two FFT operations to estimate the cross-spectrum, some multiplications for the weighting process, and an IFFT operation for computing the GCC function. If the Fourier transform of a realvalued series of length $K$ is computed using the FFT routine devised by [61], it requires $(K / 2) \log _{2}(K)-5 K / 4$ multiplications. An IFFT operation of a complex-valued series of length $K$ requires $2 K \log _{2}(K)-7 K+12$. The complexity of the PHAT algorithm is summarized in Algorithm 1. Similarly, the computational load for other GCC member algorithms can be easily counted, which will not be presented here.

Unlike the GCC method, which estimates the time delay on a frame-by-frame basis, the LMS-type adaptive algorithm updates the cost function whenever a new data sample is available. For each data sample, the number of multiplications required for computing the cost function is shown in Algorithm 2, which is higher than that of the PHAT algorithm.

The MCCC can be computed either on a block-by-block basis or in an iterative way. Its complexity is described in Algorithm 3. We see that, depending on the number of sensors, the MCCC algorithm is generally more computationaly expensive than the GCC method. Notice that more computationally efficient algorithm can be formulated to calculate MCCC using FFT. This is, however, beyond the scope of this paper.

The computational burdens required for the estimation of channel impulse responses using either the AED or the NMCFLMS algorithms are presented in Algorithms 4 and 5, respectively. Depending on the length of the modeling filter, the estimation of channel impulse responses usually requires more multiplications than estimating the generalizing crosscorrelation function. However, such a magnitude of computational complexity should not be a big concern with today's computer processors. 


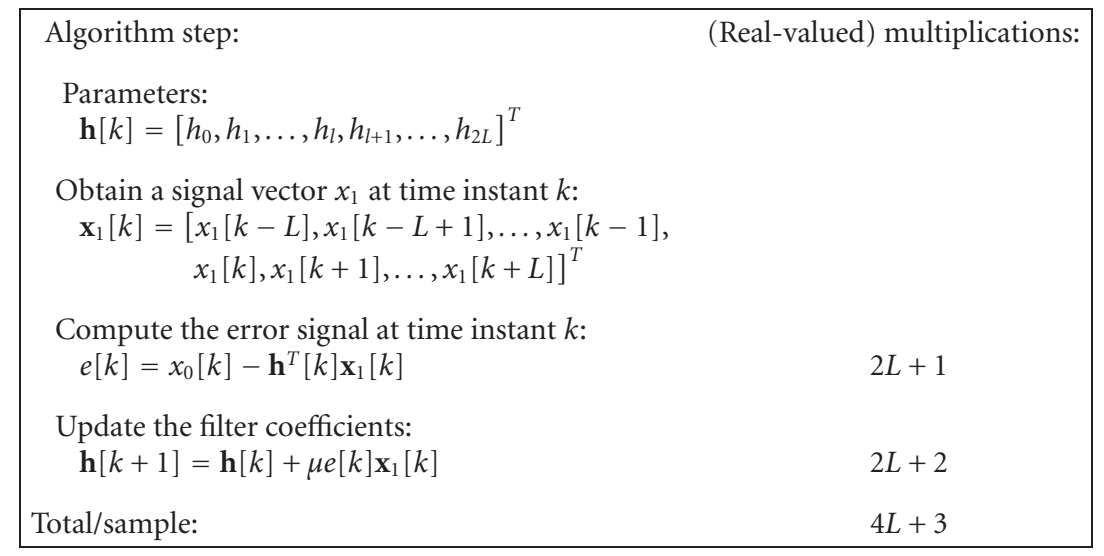

Algorithm 2: Computational complexity of the LMS-type adaptive algorithm.

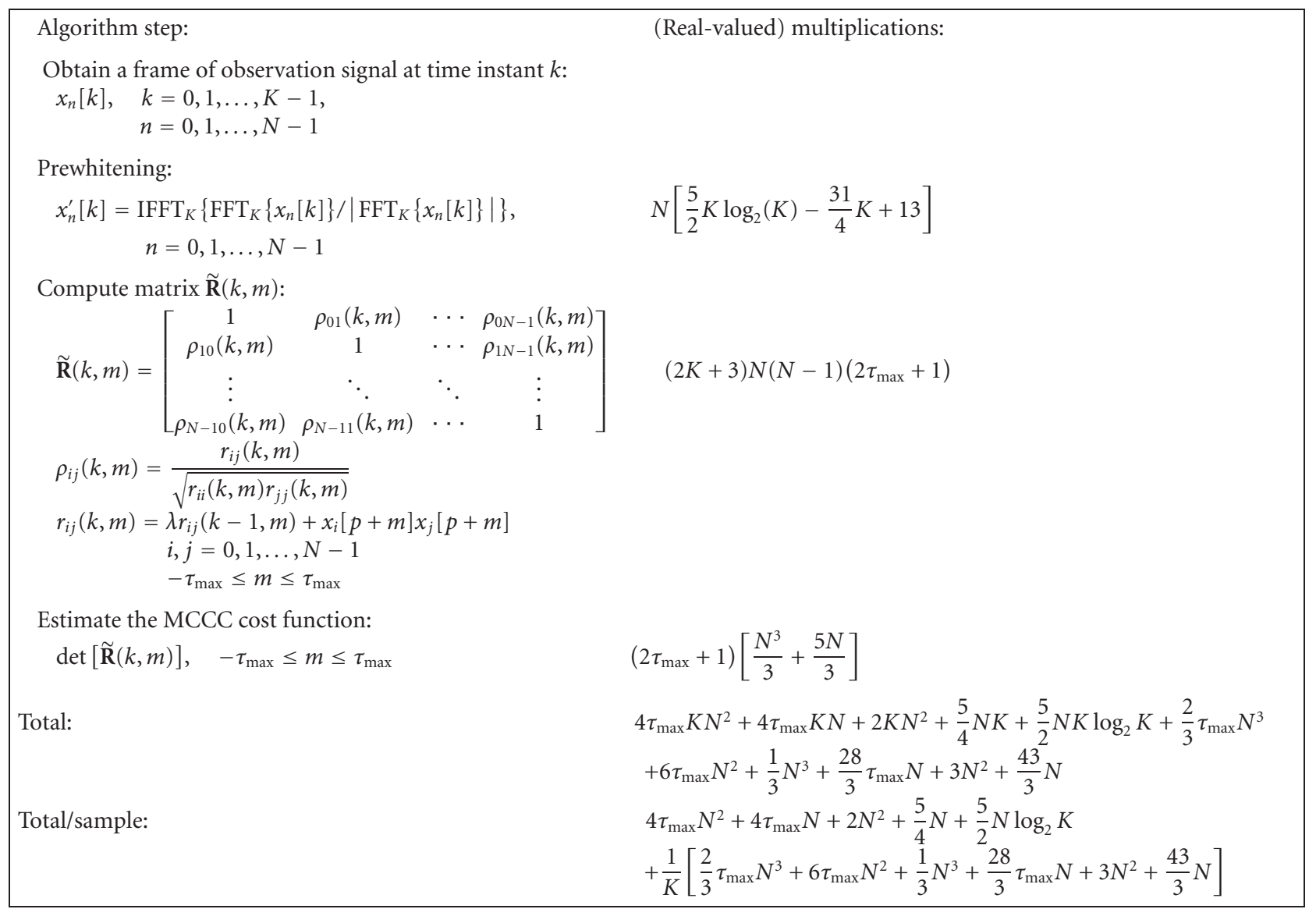

Algorithm 3: Computational complexity of the MCCC algorithm. It is assumed that determinant of a matrix is computed through LU decomposition, which requires $N^{3} / 3+5 N / 3$ multiplications [62].

\section{RESOLUTION PROBLEM}

All the TDE techniques described above measure time delay based on discrete signal samples. The delay estimate is, therefore, an integral multiple of the sampling period. Such a resolution, depending on the sampling rate and several other factors, may not be adequate for some applications. How to improve the TDE resolution becomes another challenging problem, and has attracted much attention in the past few decades. Different solutions can be applied, depending on 


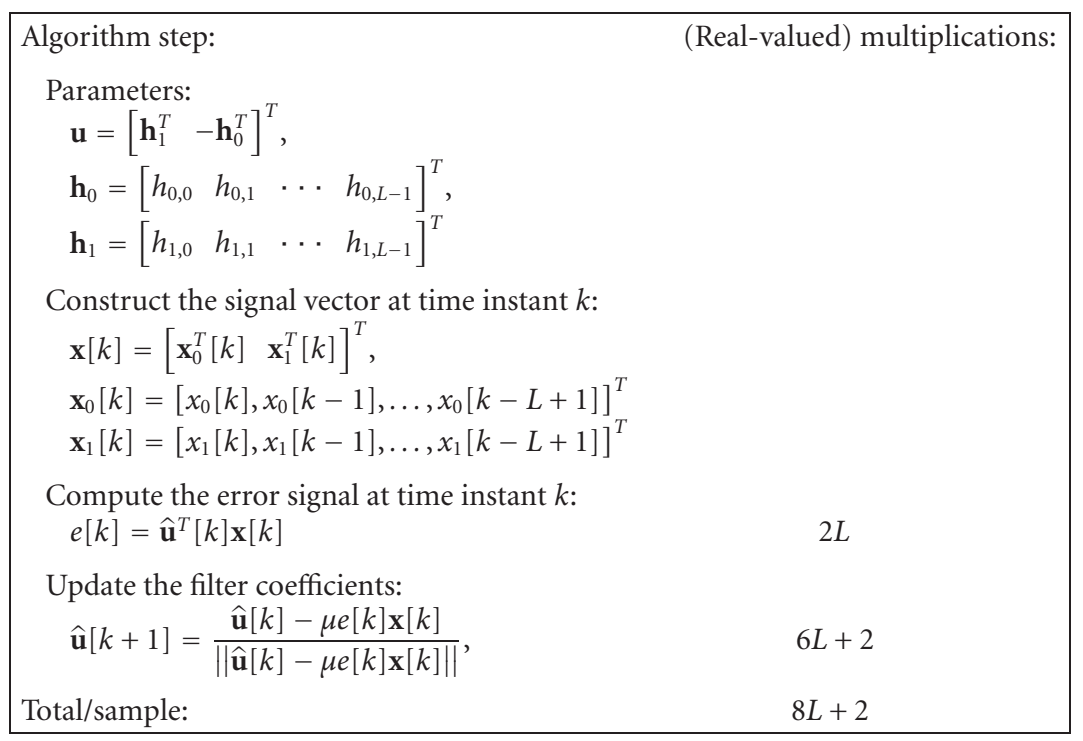

Algorithm 4: Computational complexity of the AED algorithm.

the TDE algorithm and the nature of application. To illustrate, let us examine a simple case in the context of direction of arrival (DOA) estimation, where we have two sensors and one source in the far field as shown in Figure 3. The angular resolution, which governs the ability of the system to separate two closely spaced sources, is determined by how many different DOA measurements can be made between 0 and $\pi$. Assuming that the distance between two sensors is $d$, the velocity of wave propagation is $c$, and the sampling rate is $f$, we can easily check that the maximum $\tau$ in samples that can be estimated is $d f / c$, the minimal $\tau$ is $-d f / c$, and the bearing angle $\theta$ relates to the time delay $\tau$ by

$$
\theta=\arccos \frac{c \tau}{d}
$$

Therefore, the number of different measurements of $\theta$ in $[0, \pi]$ depends on the number of different delay estimates in $[-d f / c, d f / c]$. As a result, to increase the angular resolution, we need to have more different delay measurements between $-d f / c$ and $d f / c$. This can be achieved through the following three ways.

(i) Interpolation. Since its mathematical expectation is shown to be band limited and present a symmetric peak around the true time delay, the estimated crosscorrelation function can be approximated by a concave parabola in the neighborhood of its maximum $[40,63,64]$. As a result, parabolic interpolation can be applied to the cross-correlation-based algorithms to obtain a finer TDE resolution, which is a fraction of the sampling period. Such a scheme has been adopted in many systems. However, if the statistic of the cost function is not band limited, we, in general, cannot apply parabolic interpolation. Note that in real environments, the applicability of interpolation is also limited by the SNR condition. If the SNR is very low, then interpolation will introduce significant bias. For the channel identification TDE techniques, if the estimated channel impulse responses approximate the true ones, interpolation technique can also be applied to increase resolution. However, in most situations, the impulse responses estimated with the blind techniques are only accurate enough for identifying the direct path, but not good enough for interpolation.

(ii) Increasing the sampling rate. The higher the sampling rate, the more the number of different delay estimates can be acquired between $-d f / c$ and $d f / c$, which in turn leads to a higher DOA resolution. This approach, however, will increase the complexity of both the TDE algorithm and some subsequent processing blocks of the system.

(iii) Increasing $d$. DOA resolution can also be improved by increasing $d$. Apparently, this will increase the array size. Therefore this method is hard to implement in scenarios where the space is limited. Also, a larger $d$ may cause spatial aliasing problem, which may not be a big concern for the task of source localization, but has to be treated with great care in the context of beamforming and noise reduction. In addition, increasing $d$ may lead to a higher complexity since we may have to increase the block size to compute the cost function and search the delay estimates in a larger delay range.

\section{EXPERIMENTS}

This section attempts to compare the performance of different TDE algorithms in both noisy and reverberant environments. 


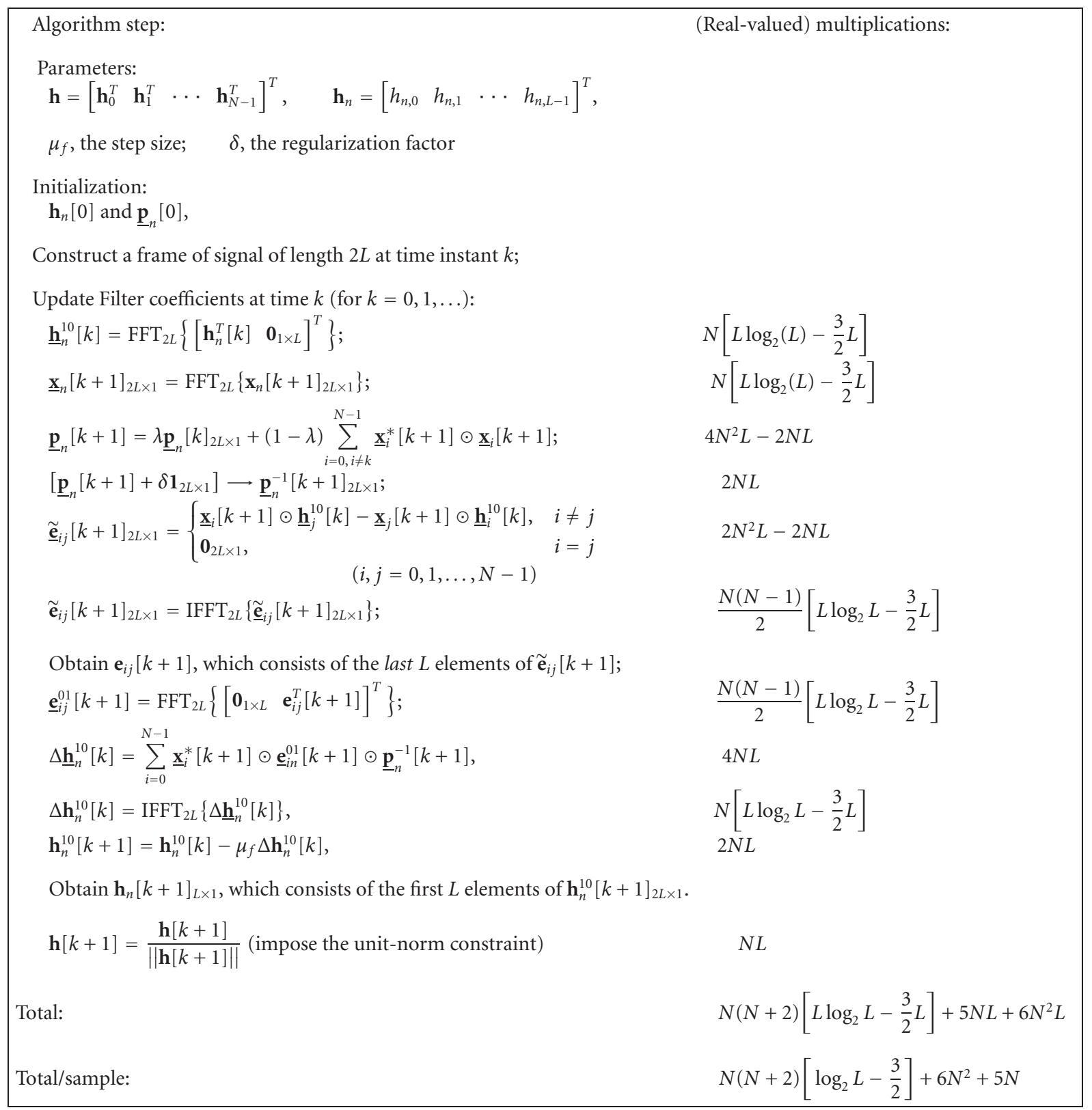

Algorithm 5: Computational complexity of the NMCFLMS algorithm. $\operatorname{FFT}_{2 L}\{\cdot\}$ and $\operatorname{IFFT}_{2 L}\{\cdot\}$ are $2 L$-point fast Fourier and inverse fast Fourier transforms, respectively. $\odot$ denotes dot product.

\subsection{Experimental setup}

In an attempt to simulate reverberant acoustic environments, the image model technology [65] is used. We consider a rectangular room with plane reflective boundaries (walls, ceiling, and floor). Each boundary is characterized by a uniform reflection coefficient, which is independent of the frequency and the incidence angle of the source signal. The following parameter values are used.

(i) Room dimensions: $120 \times 180 \times 150$ inch $(x \times y \times z)$.

(ii) Reflection coefficients: $r_{i}(i=1,2, \ldots, 6)$ varying between 0 and 1 . (iii) Source position: two point omnidirectional sources are located at $(100,100,40)$ and $(32,100,40)$, respectively.

(iv) Sensor positions: a linear array which consists of four (4) ideal point microphones placed in parallel with the $x$-axis. The four microphones are located at $(20,10$, $40),(28,10,40),(36,10,40)$, and $(44,10,40)$, respectively. The directivity pattern of each microphone is assumed to be omnidirectional.

(v) SNR: varying between $-10 \mathrm{~dB}$ and $25 \mathrm{~dB}$.

A low-pass sampled version of the impulse response of the acoustic transmission channel between each source and 
TABle 1: Parameter setup for each TDE algorithm.

\begin{tabular}{|c|c|c|c|c|c|c|}
\hline & Window size & Window type & FFT size & Smoothing factor & Filter length & Adaptation step size \\
\hline $\mathrm{CC}$ & $K=1024$ & Kaiser & $K^{\prime}=1024$ & $\gamma=0.95$ & N/A & N/A \\
\hline PHAT & $K=1024$ & Kaiser & $K^{\prime}=1024$ & $\gamma=0.95$ & N/A & N/A \\
\hline ML & $K=1024$ & Kaiser & $K^{\prime}=1024$ & $\gamma=0.95$ & N/A & N/A \\
\hline AED & $K=1024$ & Rectangular & N/A & $\gamma=0.95$ & $L=1024$ & $\mu=0.01$ \\
\hline LMS & N/A & N/A & N/A & N/A & $L=1024$ & $\mu=0.0001$ \\
\hline MCCC & $K=1024$ & Rectangular & N/A & $\gamma=0.95$ & N/A & N/A \\
\hline $\mathrm{AMC}$ & $K=1024$ & Rectangular & 2048 & $\lambda=0.8[60]$ & $L=1024$ & $\mu_{f}=0.2[60]$ \\
\hline FUSION & $K=1024$ & Kaiser & $K^{\prime}=1024$ & $\gamma=0.95$ & N/A & N/A \\
\hline
\end{tabular}

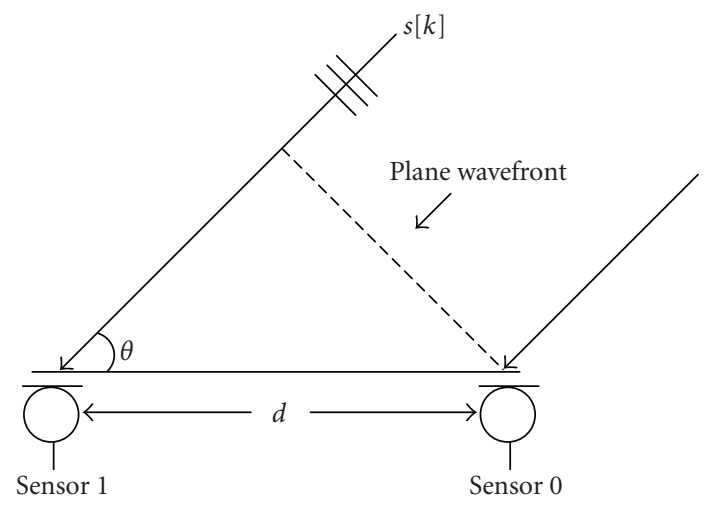

FIGURE 3: Illustration of TDE resolution problem in the context of DOA estimation.

each microphone is generated using the image method. A speech signal from a female speaker, digitized with 16-bit resolution at $16 \mathrm{kHz}$, is then convolved with the synthetic impulse responses. Finally, mutually independent white Gaussian noise is properly scaled and added to each microphone signal to control the SNR.

\subsection{Implementation}

Delay estimates were obtained on a frame-by-frame basis. The frame size used in all experiments is $64 \mathrm{~ms}$. For the crosscorrelation-based techniques (including dual- and multiplechannel algorithms), a 64-ms Kaiser window was applied to the analysis frame, while a rectangular window of the same length was applied for the channel identification-based algorithms. To reduce the temporal effect of noise on TDE performance, the cost function of each algorithm is smoothed using a single-pole recursion as follows:

$$
\bar{\Psi}_{k}=\gamma \bar{\Psi}_{k-1}+(1-\gamma) \hat{\Psi}_{k}
$$

where $\hat{\Psi}_{k}$ denotes the cost function estimated using the $k$ th frame of observation data, $\bar{\Psi}_{k}$ is a smoothed version of the cost function, based on which the delay estimates were obtained. For the MCCC algorithm, the signal was prewhitened before computing the cost function. Therefore, this method, in the case of two sensors, is equivalent to the PHAT algorithm. For the ML method, we assume that the noise spectrum is known a priori. The fusion algorithm implemented here is the consistency method presented in [51]. All the parameters used in each algorithm are summarized in Table 1.

It is not always easy to compare fairly different algorithms. In our experiments, we optimized each individual algorithm in a nonreverberant and weak noisy (SNR = $25 \mathrm{~dB}$ ) environment to its best performance. We then test and compare all the algorithms in reverberation and different noise conditions. Such a process should, in generally, not favor any specific algorithm.

\subsection{Experimental results}

A great deal of efforts have been devoted to analyzing the TDE performance of the GCC technique in reverberant environments $[66,67]$; but not much comparison has been made between correlation and system-identification-based algorithms. In this experiment, we compare all the algorithms 

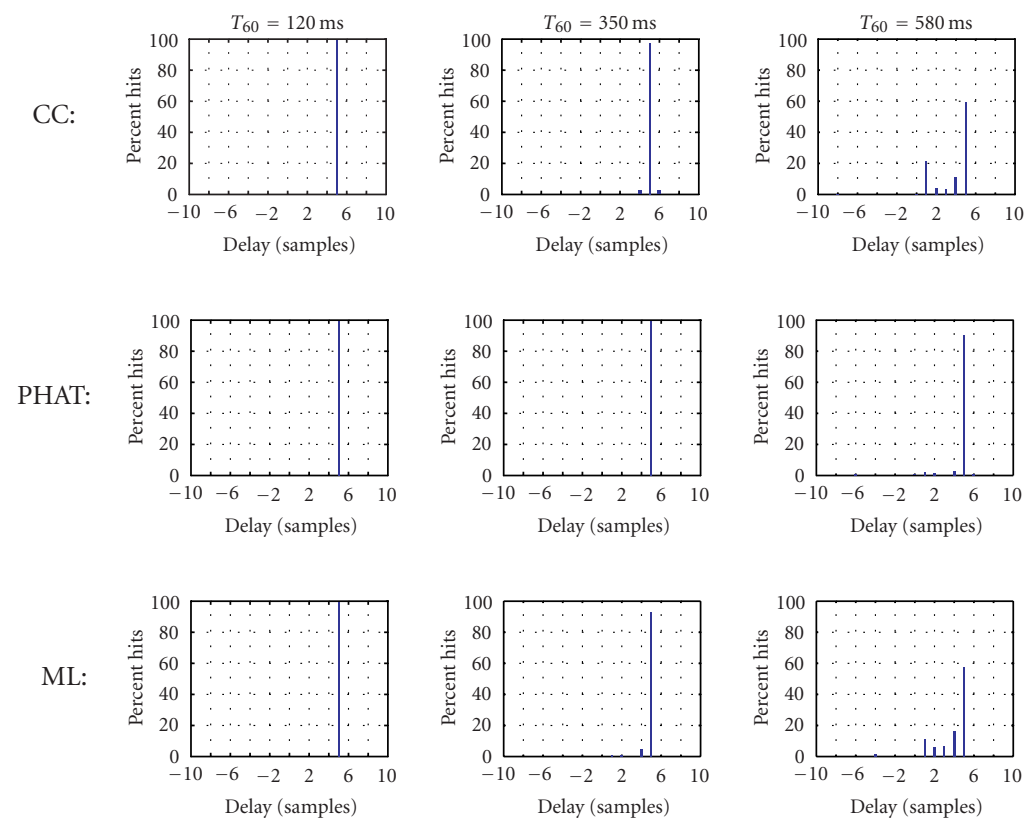

AED:
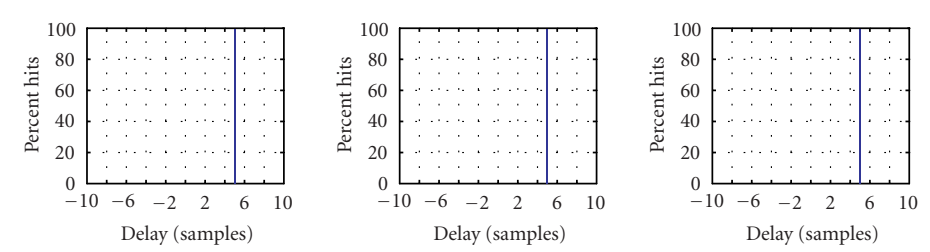

LMS:
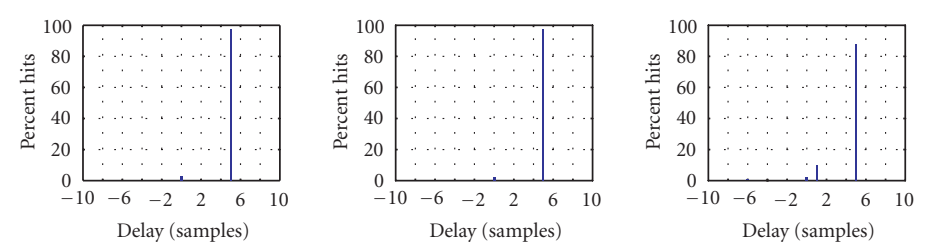

MCCC:
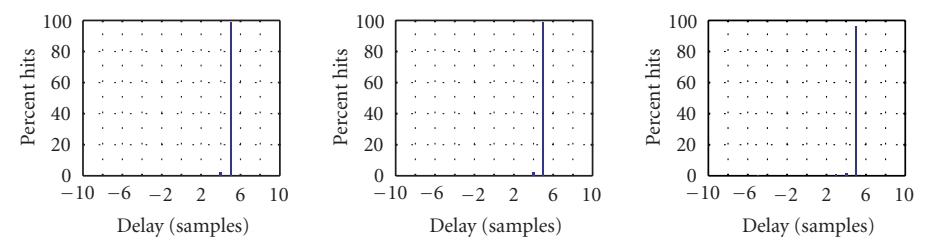

AMC:
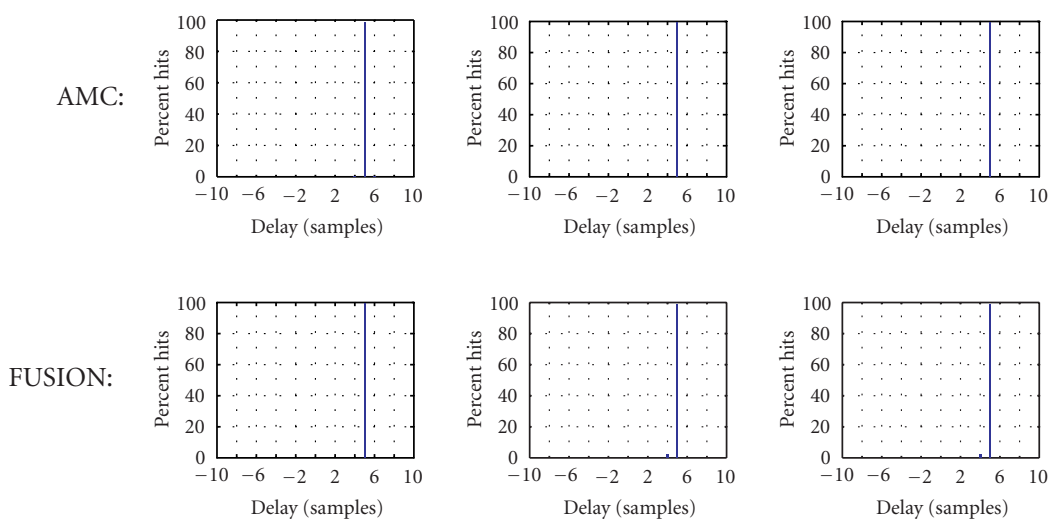

FIGURE 4: TDE performances in moderate noisy and reverberant environments, where $\mathrm{SNR}=15 \mathrm{~dB}$ and $T_{60}=120 \mathrm{~ms}, 350 \mathrm{~ms}$, and $580 \mathrm{~ms}$, respectively. 
outlined previously for their performances in different reverberant environments. Figure 4 shows histograms of TDE in a moderate noise condition where SNR $=15 \mathrm{~dB}$. The source is a speech signal from a female speaker and its location is in $(100,100,40)$. The first, second, and third columns correspond, respectively, to reverberation times of $120 \mathrm{~ms}$, $350 \mathrm{~ms}$, and $580 \mathrm{~ms}$. The true time delay between sensor 0 and 1 is equal to 5 (samples). It can be seen that, in the first two reverberant environments, all algorithms can accurately identify the time delay. When reverberation time is increased to $580 \mathrm{~ms}$, both the CC and the ML methods suffer significant performance degradation, showing that these two approaches are sensitive to reverberation. The PHAT algorithm, though also belongs to the GCC family like the CC and ML methods, still yields a reasonable performance, implying its robustness with respect to reverberation. This corroborates with many observations reported in the literature [46]. Among the five techniques that use two sensors (i.e., CC, PHAT, ML, AED, LMS), the AED algorithm delivers the best performance. This indicates that taking it into account in the signal model is an effective way in dealing with reverberation. Comparing the MCCC, AMC, and fusion algorithms with dual-sensor techniques, one can easily see the advantage of using multiple sensors. Since the AMC algorithm was formulated from the reverberation signal model and using multiple sensors, it is not surprising to see that it achieves the best performance in this strong reverberant environment.

The second experiment involves a set of data obtained in nonreverberant (simulated by setting all the reflection coefficients to 0 ) but noisy environments. The source signal and its presentation are the same as in the previous experiment. Figure 5 shows histograms of delay estimates. The first, second, and third columns correspond, respectively, to noise conditions of $\mathrm{SNR}=15 \mathrm{~dB}, 5 \mathrm{~dB}$, and $-5 \mathrm{~dB}$. In general, all TDE techniques are quite robust to noise. They work pretty well even when SNR is as low as $5 \mathrm{~dB}$. When SNR drops down to $-5 \mathrm{~dB}$, the TDE performance begin to deteriorate, even though the degree of degradation may vary across algorithms. Among all the eight algorithms studied, the LMS method is most sensitive to noise. We may consider to improve this technique by using some adaptive algorithms that have a faster convergence rate or a low steady-state error. The PHAT algorithm, which demonstrated the highest robustness with respect to reverberation in the GCC family, is inferior to both the CC and the ML approaches in additive noise. The ML algorithm delivers a better performance than the CC method. This indicates that some a priori knowledge can help the estimator to cope with distortion. Among the five dual-sensor techniques, we noticed that the AED algorithm demonstrates the highest robustness not only to reverberation, but to additive noise as well. This observation is different from our intuition since it is well perceived that the blind channel identification technique is in general sensitive to noise. We attribute this to the nature of the TDE problem, which only requires to identify the direct path. Estimation of the whole impulse response, depending on its length and many other factors, may be sensitive to noise; but identification of the direct path is a much easier task, and it can be immune to noise.

Comparing the AMC with the AED algorithm, we did not see much improvement as we observed in the previous experiment. This is understandable. The motivation behind the AMC algorithm is to circumvent the common-zero problem. The probability of a common zero shared among channels decreases when the number of channels increases. However, in this experiment, all the channels apparently share no common zero since there is no reverberation. As a result, the AMC is similar to the AED algorithm in performance.

Both the MCCC and fusion methods yield a performance superior to that of the techniques with two sensors, indicating that using multiple sensors is a good way to improve the robustness of TDE with respect to additive noise. The MCCC shows a better performance than the fusion method.

The final experiment is to test the TDE algorithms for their tracking ability. To simulate a moving source, we first place the source at $(100,100,40)$ for 30 seconds, and then switch to $(32,100,40)$. Again, the source is a speech signal as used in the previous experiments. In this case, the true delay in the first 30 seconds is 5 (samples), and 3 (samples) then. The average SNR is $0 \mathrm{~dB}$, and $T_{60}=240 \mathrm{~ms}$. Figure 6 shows the TDE results. The AED, LMS, and AMC algorithms are adaptive in nature. They take some time to converge to a new delay. All other five methods are nonadaptive. However, due to the smoothing processing, they also take some time to adapt to the new source position. From the results, one can see that all the algorithms can adjust to the new delay in less than one second.

\section{SUMMARY}

Time delay estimation, which serves as a fundamental step for a source localization or a beamforming system, has attracted a considerable amount of research attention in the past few decades. Various techniques were developed in the literature. This paper briefly summarized these efforts, and reviewed the state of the art, the critical techniques, and the recent advances which had significantly improved performance of time delay estimation in adverse environments. Broadly, the reviewed techniques can be classified into two categories: cross-correlation-based methods and system identification-based approaches. Both categories can be implemented either based on two sensors, or using multiple sensors. We evaluated eight algorithms, including five dualchannel techniques and three multiple-channel techniques, in both reverberant and noisy environments. Among the five studied dual-channel techniques, the adaptive eigenvalue decomposition algorithm demonstrated the best performance in both noise and reverberation conditions, showing its great potential for real applications. In general, more sensors will lead to a higher robustness because of the redundancy. However, it should be pointed out that attention has to be paid to implementing the multichannel cross-correlation algorithm and the fusion method. Both need to synchronize either the signals observed at different sensors, or the cost functions from different sensor pairs. In case that the true delay is not 

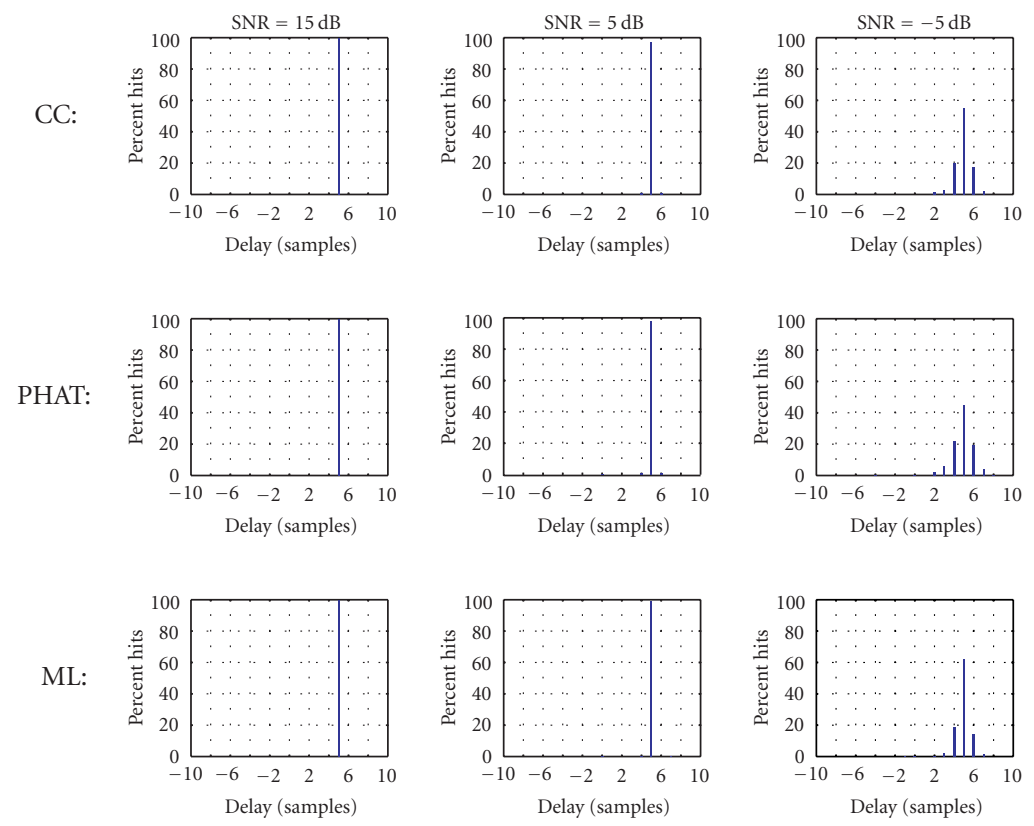

AED:
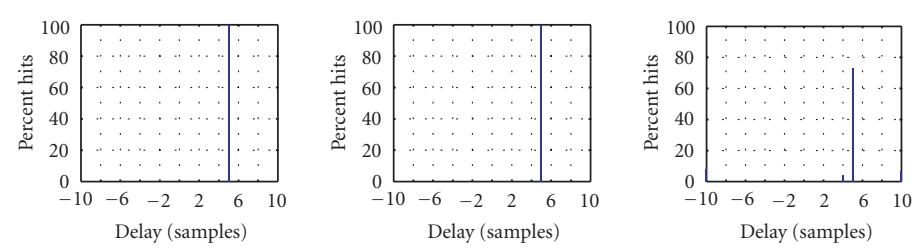

LMS:
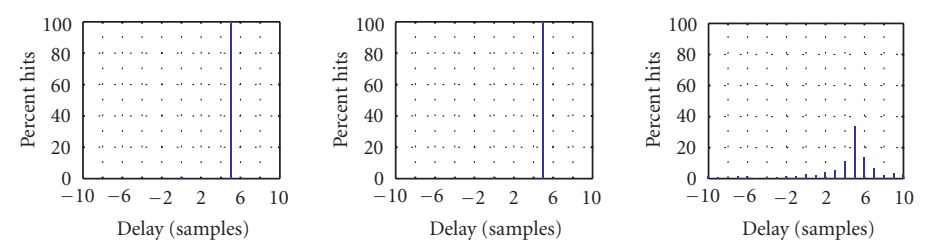

MCCC:
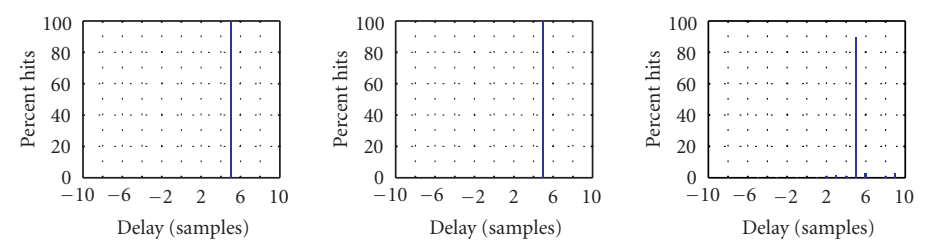

AMC:
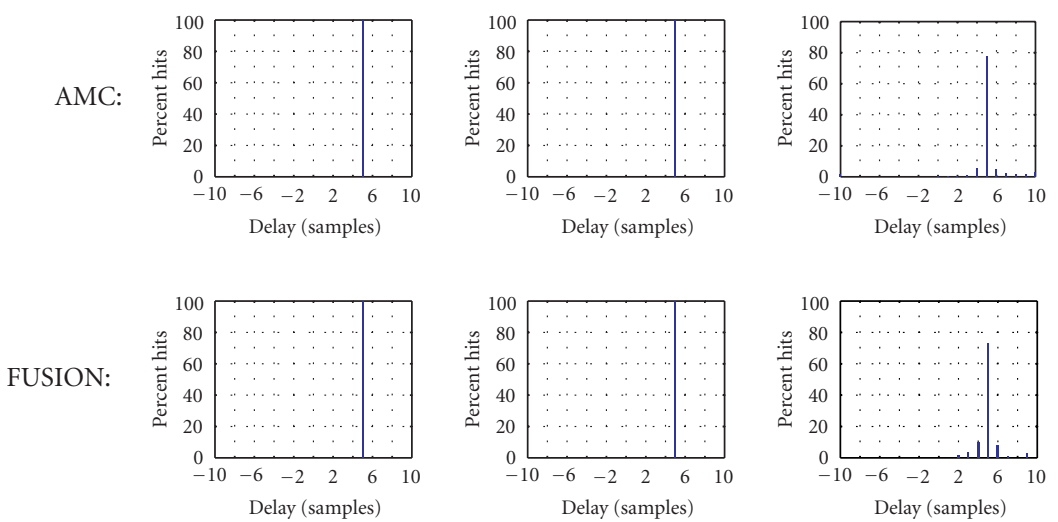

FIGURE 5: TDE performances in nonreverberant but noisy environments, where SNR $=15 \mathrm{~dB}, 5 \mathrm{~dB}$, and $-5 \mathrm{~dB}$, respectively. 

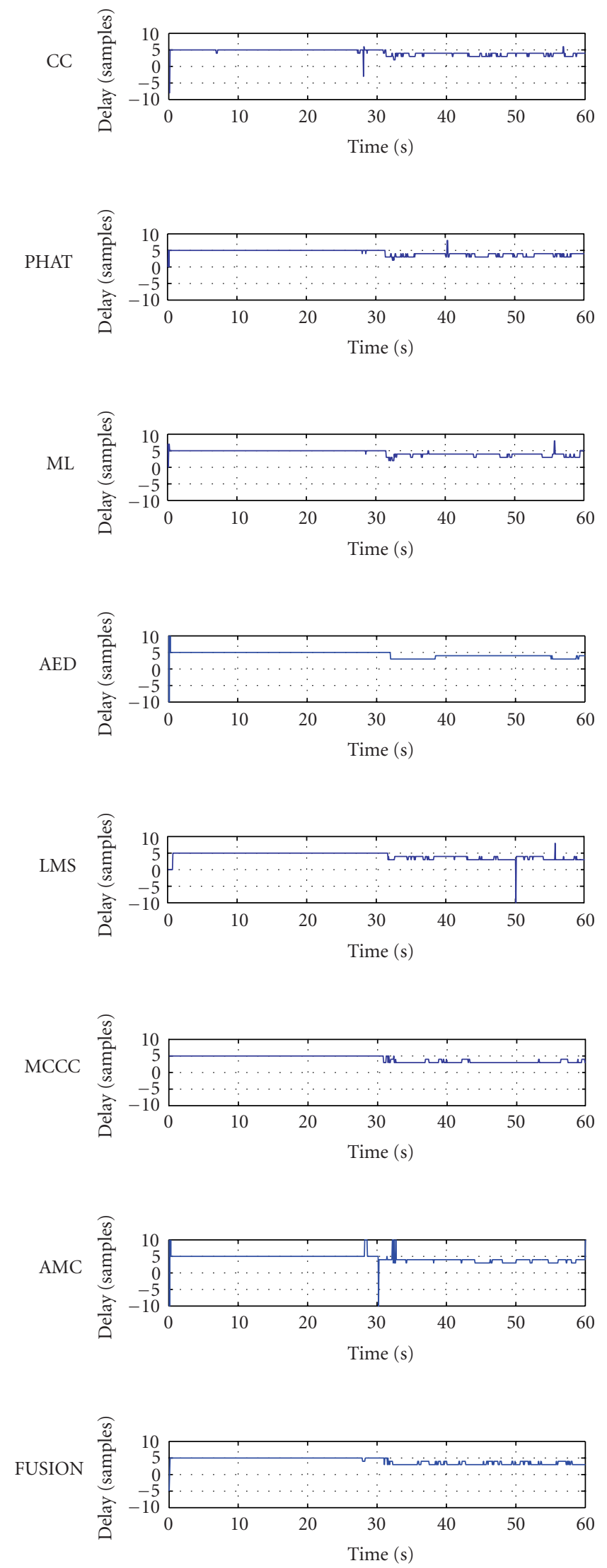

FIGURE 6: Tracking performance of different algorithms in a noisy and reverberant environment, where $\mathrm{SNR}=0 \mathrm{~dB}$ and $T_{60}=240 \mathrm{~ms}$. 
the integral multiple of the sampling rate, we will have to either increase the sampling rate or use interpolation, which may significantly increase the computational complexity. In case that the observation signals or the cost functions are not properly aligned, we may not achieve much improvement.

\section{REFERENCES}

[1] J. E. Ehrenberg, T. E. Ewart, and R. D. Morris, "Signalprocessing techniques for resolving individual pulses in a multipath signal," Journal of the Acoustical Society of America, vol. 63, no. 6, pp. 1861-1865, 1978.

[2] N. L. Owsley and G. R. Swope, "Time delay estimation in a sensor array," IEEE Transactions on Acoustics, Speech, and Signal Processing, vol. 29, no. 3, pp. 519-523, 1981.

[3] R. J. Tremblay, G. C. Carter, and D. W. Lytle, "A practical approach to the estimation of amplitude and time-delay parameters of a composite signal," IEEE Journal of Oceanic Engineering, vol. 12, no. 1, pp. 273-278, 1987.

[4] R. Wu, J. Li, and Z.-S. Liu, "Super resolution time delay estimation via MODE-WRELAX," IEEE Transactions on Aerospace and Electronic Systems, vol. 35, no. 1, pp. 294-307, 1999.

[5] C. H. Knapp and G. C. Carter, "The generalized correlation method for estimation of time delay," IEEE Transactions on Acoustics, Speech, and Signal Processing, vol. 24, no. 4, pp. 320327, 1976.

[6] G. C. Carter, "Time delay estimation for passive sonar signal processing," IEEE Transactions on Acoustics, Speech, and Signal Processing, vol. 29, no. 3, pp. 463-470, 1981.

[7] G. C. Carter, "Coherence and time delay estimation," in Signal Processing Handbook, C. H. Chen, Ed., pp. 443-482, Marcel Dekker, New York, NY, USA, 1988.

[8] A. H. Quazi, "An overview on the time delay estimate in active and passive systems for target localization," IEEE Transactions on Acoustics, Speech, and Signal Processing, vol. 29, no. 3, pp. 527-533, 1981.

[9] G. C. Carter, Ed., Coherence and Time Delay Estimation: An Applied Tutorial for Research, Development, Test and Evaluation Engineers, IEEE Press, New York, NY, USA, 1993.

[10] M. Feder and E. Weinstein, "Parameter estimation of superimposed signals using the EM algorithm," IEEE Transactions on Acoustics, Speech, and Signal Processing, vol. 36, no. 4, pp. 477-489, 1988.

[11] G. Su and M. Morf, "The signal subspace approach for multiple wide-band emitter location," IEEE Transactions on Acoustics, Speech, and Signal Processing, vol. 31, no. 6, pp. 15021522, 1983.

[12] S. S. Reddi, "Multiple source location-a digital approach," IEEE Transactions on Aerospace and Electronic Systems, vol. 15, no. 1, pp. 95-105, 1979.

[13] T. G. Manickam, R. J. Vaccaro, and D. W. Tufts, "A leastsquares algorithm for multipath time-delay estimation," IEEE Transactions on Signal Processing, vol. 42, no. 11, pp. 32293233, 1994.

[14] J.-J. Fuchs, "Multipath time-delay detection and estimation," IEEE Transactions on Signal Processing, vol. 47, no. 1, pp. 237243, 1999.

[15] J. Benesty, "Adaptive eigenvalue decomposition algorithm for passive acoustic source localization," Journal of the Acoustical Society of America, vol. 107, no. 1, pp. 384-391, 2000.

[16] S. Doclo and M. Moonen, "Robust adaptive time delay estimation for speaker localization in noisy and reverberantacoustic environments," EURASIP Journal on Applied Signal Processing, vol. 2003, no. 11, pp. 1110-1124, 2003.

[17] T. G. Dvorkind and S. Gannot, "Approaches for time different of arrival estimation in a noisy and reververant environment," in Proceedings of International Workshop on Acoustic Echo and Noise Control (IWAENC '03), pp. 215-218, Kyoto, Japan, September 2003.

[18] J. C. Hassab and R. E. Boucher, "Performance of the generalized cross correlator in the presence of a strong spectral peak in the signal," IEEE Transactions on Acoustics, Speech, and Signal Processing, vol. 29, no. 3, pp. 549-555, 1981.

[19] L. E. Miller and J. S. Lee, "Error analysis of time delay estimation using a finite integration time correlator," IEEE Transactions on Acoustics, Speech, and Signal Processing, vol. 29, no. 3, pp. 490-496, 1981.

[20] J. P. Ianniello, "Time delay estimation via cross-correlation in the presence of large estimation errors," IEEE Transactions on Acoustics, Speech, and Signal Processing, vol. 30, no. 6, pp. 9981003, 1982.

[21] M. Azaria and D. Hertz, "Time delay estimation by generalized cross correlation methods," IEEE Transactions on Acoustics, Speech, and Signal Processing, vol. 32, no. 2, pp. 280-285, 1984.

[22] Y. Bar-Shalom, F. Palmieri, A. Kumar, and H. M. Shertukde, "Analysis of wide-band cross correlation for time-delay estimation," IEEE Transactions on Signal Processing, vol. 41, no. 1, pp. 385-387, 1993.

[23] J. K. Tugnait, "Time delay estimation with unknown spatially correlated Gaussian noise," IEEE Transactions on Signal Processing, vol. 41, no. 2, pp. 549-558, 1993.

[24] Y. Wu, "Time delay estimation of non-Gaussian signal in unknown Gaussian noises using third-order cumulants," Electronics Letters, vol. 38, no. 16, pp. 930-931, 2002.

[25] Y. (Arden) Huang and J. Benesty, "A class of frequency-domain adaptive approaches to blind multichannel identification," IEEE Transactions on Signal Processing, vol. 51, no. 1, pp. 1124, 2003.

[26] F. A. Reed, P. L. Feintuch, and N. J. Bershad, "Time delay estimation using the LMS adaptive filter-static behavior," IEEE Transactions on Acoustics, Speech, and Signal Processing, vol. 29, no. 3, pp. 561-571, 1981.

[27] D. M. Etter and S. D. Stearns, "Adaptive estimation of time delays in sampled data systems," IEEE Transactions on Acoustics, Speech, and Signal Processing, vol. 29, no. 3, pp. 582-587, 1981.

[28] D. H. Youn, N. Ahmed, and G. C. Carter, "On using the LMS algorithm for time delay estimation," IEEE Transactions on Acoustics, Speech, and Signal Processing, vol. 30, no. 5, pp. 798801, 1982.

[29] P. C. Ching and Y. T. Chan, "Adaptive time delay estimation with constraints," IEEE Transactions on Acoustics, Speech, and Signal Processing, vol. 36, no. 4, pp. 599-602, 1988.

[30] H. C. So, P. C. Ching, and Y. T. Chan, "A new algorithm for explicit adaptation of time delay," IEEE Transactions on Signal Processing, vol. 42, no. 7, pp. 1816-1820, 1994.

[31] P. P. Moghaddam, H. Amindavar, and R. L. Kirlin, "A new time-delay estimation in multipath," IEEE Transactions on Signal Processing, vol. 51, no. 5, pp. 1129-1142, 2003.

[32] J. P. Ianniello, "Large and small error performance limits for multipath time delay estimation," IEEE Transactions on Acoustics, Speech, and Signal Processing, vol. 34, no. 2, pp. 245-251, 1986.

[33] J. C. Hassab, "Contact localization and motion analysis in the ocean environment: a perspective," IEEE Journal of Oceanic Engineering, vol. 8, no. 3, pp. 136-147, 1983. 
[34] F. El-Hawary, F. Aminzadeh, and G. A. N. Mbamalu, "The generalized Kalman filter approach to adaptive underwater target tracking," IEEE Journal of Oceanic Engineering, vol. 17, no. 1, pp. 129-137, 1992.

[35] C. S. Clay and H. Medwin, Acoustical Oceanography, John Wiley \& Sons, New York, NY, USA, 1977.

[36] A. Stéphenne and B. Champagne, "Cepstral prefiltering for time delay estimation in reverberant environments," in Proceedings of IEEE International Conference on Acoustics, Speech, and Signal Processing (ICASSP'95), vol. 5, pp. 3055-3058, Detroit, Mich, USA, May 1995.

[37] M. S. Brandstein and H. F. Silverman, "A robust method for speech signal time-delay estimation in reverberant rooms," in Proceedings of IEEE International Conference on Acoustics, Speech, and Signal Processing (ICASSP '97), vol. 1, pp. 375-378, Munich, Germany, April 1997.

[38] T. G. Dvorkind and S. Gannot, "Time difference of arrival estimation of speech source in a noisy and reverberant environment," Signal Processing, vol. 85, no. 1, pp. 177-204, 2005.

[39] Y. (Arden) Huang and J. Benesty, "Adaptive multichannel time delay estimation based on blind system identification for acoustic source localization," in Adaptive Signal ProcessingApplications to Real-World Problems, J. Benesty and Y. (Arden) Huang, Eds., chapter 8, pp. 227-248, Springer, Berlin, Germany, 2003.

[40] G. Jacovitti and G. Scarano, "Discrete time techniques for time delay estimation," IEEE Transactions on Signal Processing, vol. 41, no. 2, pp. 525-533, 1993.

[41] G. Jacovitti, A. Neri, and R. Cusani, "On a fast digital method of estimating the autocorrelation of a Gaussian stationary process," IEEE Transactions on Acoustics, Speech, and Signal Processing, vol. 32, no. 5, pp. 968-976, 1984.

[42] G. Jacovitti and R. Cusani, "An efficient technique for high correlation estimation," IEEE Transactions on Acoustics, Speech, and Signal Processing, vol. 35, no. 5, pp. 654-660, 1987.

[43] J. Chen, J. Benesty, and Y. (Arden) Huang, "Performance of GCC- and AMDF-based time-delay estimation in practical reverberant environments," EURASIP Journal on Applied Signal Processing, vol. 2005, no. 1, pp. 25-36, 2005.

[44] G. C. Carter, A. H. Nuttall, and P. G. Cable, "The smoothed coherence transform," Proceedings of the IEEE, vol. 61, no. 10, pp. 1497-1498, 1973.

[45] P. R. Roth, "Effective measurements using digital signal analysis,” IEEE Spectrum, vol. 8, no. 4, pp. 62-70, 1971.

[46] H. Wang and P. Chu, "Voice source localization for automatic camera pointing system in video conferencing," in Proceedings of IEEE International Conference on Acoustics, Speech, and Signal Processing (ICASSP '97), vol. 1, pp. 187-190, Munich, Germany, April 1997.

[47] P. L. Feintuch, N. J. Bershad, and F. A. Reed, "Time delay estimation using the LMS adaptive filter-dynamic behavior," IEEE Transactions on Acoustics, Speech, and Signal Processing, vol. 29, no. 3, pp. 571-576, 1981.

[48] S. Haykin, "Radar array processing for angle of arrival estimation," in Array Signal Processing, S. Haykin, Ed., pp. 194-292, Prentice-Hall, Englewood Cliffs, NJ, USA, 1985.

[49] R. L. Kirlin, D. F. Moore, and R. F. Kubichek, "Improvement of delay measurements from sonar arrays via sequential state estimation," IEEE Transactions on Acoustics, Speech, and Signal Processing, vol. 29, no. 3, pp. 514-519, 1981.

[50] T. Nishiura, T. Yamada, S. Nakamura, and K. Shikano, "Localization of multiple sound sources based on a CSP analysis witha microphone array," in Proceedings of IEEE International Conference on Acoustics, Speech, and Signal Processing (ICASSP '00), vol. 2, pp. 1053-1055, Istanbul, Turkey, June 2000.
[51] S. M. Griebel and M. S. Brandstein, "Microphone array source localization using realizable delay vectors," in Proceedings of IEEE Workshop on the Applications of Signal Processing to Audio and Acoustics (WASPAA '01), pp. 71-74, New Platz, NY, USA, October 2001.

[52] J. H. DiBiase, H. F. Silverman, and M. S. Branstein, "Robust localization in reverberant rooms," in Microphone Arrays: Signal Processing Techniques and Applications, M. S. Branstein and D. B. Ward, Eds., chapter 8, pp. 157-180, Springer, New York, NY, USA, 2001.

[53] J. Chen, J. Benesty, and Y. (Arden) Huang, "Robust time delay estimation exploiting redundancy among multiple microphoens," IEEE Transactions on Speech and Audio Processing, vol. 11, no. 6, pp. 549-557, 2003.

[54] J. Benesty, J. Chen, and Y. (Arden) Huang, "Time-delay estimation via linear interpolation and cross correlation," IEEE Transactions on Speech and Audio Processing, vol. 12, no. 5, pp. 509-519, 2004.

[55] Y. (Arden) Huang, J. Benesty, and G. W. Elko, "Adaptive eigenvalue decomposition algorithm for real time acoustic source localization system," in Proceedings of IEEE International Conference on Acoustics, Speech, and Signal Processing (ICASSP '99), vol. 2, pp. 937-940, Phoenix, Ariz, USA, March 1999.

[56] G. Xu, H. Liu, L. Tong, and T. Kailath, "A least-squares approach to blind channel identification," IEEE Transactions on Signal Processing, vol. 43, no. 12, pp. 2982-2993, 1995.

[57] H.-F. Chen, X.-R. Cao, and J. Zhu, "Convergence of stochasticapproximation-based algorithms for blind channel identification," IEEE Transactions on Information Theory, vol. 48, no. 5, pp. 1214-1225, 2002.

[58] M. İ. Gürelli and C. L. Nikias, "EVAM: an eigenvector-based algorithm for multichannel blind deconvolution of input colored signals," IEEE Transactions on Signal Processing, vol. 43, no. 1, pp. 134-149, 1995.

[59] L. Tong and S. Perreau, "Multichannel blind identification: from subspace to maximum likelihood methods," Proceedings of the IEEE, vol. 86, no. 10, pp. 1951-1968, 1998.

[60] Y. (Arden) Huang and J. Benesty, "Adaptive multi-channel least mean square and Newton algorithms for blind channel identification," Signal Processing, vol. 82, no. 8, pp. 1127-1138, 2002.

[61] H. V. Sorensen, D. L. Jones, M. T. Heideman, and C. S. Burrus, "Real-valued fast Fourier transform algorithms," IEEE Transactions on Acoustics, Speech, and Signal Processing, vol. 35, no. 6, pp. 849-863, 1987.

[62] L. Fox, An Introduction to Numerical Linear Algebra, Clarendon Press, Oxford, UK, 1964.

[63] R. E. Boucher and J. C. Hassab, "Analysis of discrete implementation of generalized cross correlator," IEEE Transactions on Acoustics, Speech, and Signal Processing, vol. 29, no. 3, pp. 609-611, 1981.

[64] R. Moddemeijer, "On the determination of the position of extrema of sampled correlators," IEEE Transactions on Signal Processing, vol. 39, no. 1, pp. 216-219, 1991.

[65] J. B. Allen and D. A. Berkley, "Image method for efficiently simulating small-room acoustics," Journal of the Acoustical Society of America, vol. 65, no. 4, pp. 943-950, 1979.

[66] B. Champagne, S. Bedard, and A. Stephenne, "Performance of time-delay estimation in the presence of room reverberation," IEEE Transactions on Speech and Audio Processing, vol. 4, no. 2, pp. 148-152, 1996.

[67] T. Gustafsson, B. D. Rao, and M. Trivedi, "Source localization in reverberant environments: modeling and statistical 
analysis," IEEE Transactions on Speech and Audio Processing, vol. 11, no. 6, pp. 791-803, 2003.

Jingdong Chen received the B.S. degree in electrical engineering and the M.S. degree in array signal processing from the Northwestern Polytechnic University in 1993 and 1995 respectively, and the Ph.D. degree in pattern recognition and intelligence control from the Chinese Academy of Sciences in 1998. From 1998 to 1999, he was with ATR Interpreting Telecommunications Research Laboratories, Kyoto, Japan. He then joined

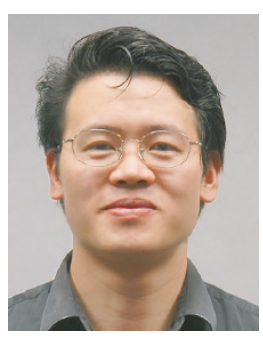
the Griffith University, Brisbane, Australia, as a Research Fellow. From 2000 to 2001, he worked at ATR Spoken Language Translation Research Laboratories, Kyoto, Japan. He joined Bell Laboratories as a Member of Technical Staff in July 2001. His research interests include adaptive signal processing, speech enhancement, adaptive noise/echo cancellation, and microphone array processing. He coauthored one monograph book and coauthored/coedited one edited book.

Jacob Benesty received the Masters degree in microwaves from Pierre and Marie Curie University, France, in 1987, and the Ph.D. degree in control and signal processing from Orsay University, France, in April 1991. From January 1994 to July 1995, he worked at Telecom Paris University. From October 1995 to May 2003, he was first a Consultant and then a Member of the Technical Staff at Bell Laboratories, Murray Hill,

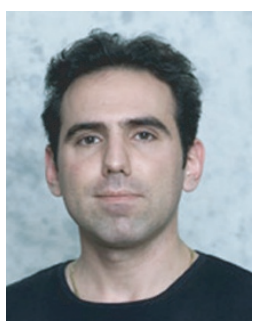
NJ, USA. In May 2003, he joined the University of Quebec, INRSEMT, in Montreal, Quebec, Canada, as an Associate Professor. His research interests are in acoustic signal processing and multimedia communications. Dr. Benesty received the 2001 Best Paper Award from the IEEE Signal Processing Society. He was a Member of the editorial board of the EURASIP Journal on Applied Signal Processing and was the cochair of the 1999 International Workshop on Acoustic Echo and Noise Control. He coauthored two books. He also coedited/coauthored four other books.

Yiteng (Arden) Huang Huang received the B.S. degree from the Tsinghua University in 1994, the M.S. and Ph.D. degrees from the Georgia Institute of Technology (Georgia Tech) in 1998 and 2001, respectively, all in electrical and computer engineering. Now he is a Member of technical staff at Bell Labs, where he conducts research in acoustic and speech signal processing for multimedia communications. Dr. Huang is currently an Associat Editor of the EURASIP Journal on Applied Signal Processing. He is a Member of the Signal Processing Theory and Methods and the Audio and Electroacoustics Technical Committees of the IEEE Signal Processing Society. He served as an Associat Editor for the IEEE Signal Processing Letters from 2002 to 2005. He was a technical cochair of the 2005 Joint Workshop on Hands-Free Speech Communication and Microphone Array. He coauthored one monograph book and coauthored/coedited two other edited books. He received the 2002 Young Author Best Paper Award from the IEEE Signal Processing Society, and a number of other awards/honors for his academic performance and services. 